\title{
THE EVOLUTION AND ENVIRONMENTS OF X-RAY EMITTING ACTIVE GALACTIC NUCLEI IN HIGH-REDSHIFT LARGE-SCALE STRUCTURES
}

\author{
N. Rumbaugh ${ }^{1}$, D. D. Kocevssi ${ }^{2}$, R. R. Gal ${ }^{3}$, B. C. Lemaux ${ }^{1}$, L. M. Lubin ${ }^{1}$, C. D. Fassnacht ${ }^{1}$, \\ E. J. MCGRATH ${ }^{2}$, AND G. K. SQuires ${ }^{4}$ \\ ${ }^{1}$ Department of Physics, University of California-Davis, 1 Shields Avenue, Davis, CA 95616, USA; narumbaugh@ucdavis.edu \\ ${ }^{2}$ University of California Observatories/Lick Observatory, University of California, Santa Cruz, CA 95064, USA \\ ${ }^{3}$ Institute for Astronomy, University of Hawai' 1 , 2680 Woodlawn Drive, HI 96822, USA \\ ${ }^{4}$ Spitzer Space Center, California Institute of Technology, M/S 220-6, 1200 E. California Blvd., Pasadena, CA 91125, USA \\ Received 2011 August 23; accepted 2011 December 2; published 2012 February 2
}

\begin{abstract}
We use deep Chandra imaging and an extensive optical spectroscopy campaign on the Keck $10 \mathrm{~m}$ telescopes to study the properties of X-ray point sources in two isolated X-ray-selected clusters, two superclusters, and one "supergroup" at redshifts of $z \sim 0.7-0.9$. We first study X-ray point sources using the statistical measure of cumulative source counts, finding that the measured overdensities are consistent with previous results, but we recommend caution in overestimating the precision of the technique. Optical spectroscopy of objects matched to X-ray point sources confirms a total of 27 active galactic nuclei (AGNs) within 5 structures, and we find that their host galaxies tend to be located away from dense cluster cores. More than $36 \%$ of the host galaxies are located in the "green valley" on a color-magnitude diagram, which suggests they are a transitional population. Based on analysis of [O II] and $\mathrm{H} \delta$ line strengths, the average spectral properties of the AGN host galaxies in all structures indicate either ongoing star formation or a starburst within $\sim 1 \mathrm{Gyr}$, and that the host galaxies are younger than the average galaxy in the parent population. These results indicate a clear connection between starburst and nuclear activity. We use composite spectra of the spectroscopically confirmed members in each structure (cluster, supergroup, or supercluster) to separate them based on a measure of the overall evolutionary state of their constituent galaxies. We define structures as having more evolved populations if their average galaxy has lower $\mathrm{EW}([\mathrm{O} \mathrm{II}])$ and $\mathrm{EW}(\mathrm{H} \delta)$. The AGNs in the more evolved structures have lower rest-frame $0.5-8 \mathrm{keV} X$-ray luminosities (all below $10^{43.3} \mathrm{erg} \mathrm{s}^{-1}$ ) and longer times since a starburst than those in the unevolved structures, suggesting that the peak of both star formation and AGN activity has occurred at earlier times. With the wide range of evolutionary states and time frames in the structures, we use our results to analyze the evolution of X-ray AGNs and evaluate potential triggering mechanisms.
\end{abstract}

Key words: galaxies: active - galaxies: clusters: general - galaxies: evolution - galaxies: nuclei - X-rays: galaxies

Online-only material: color figures

\section{INTRODUCTION}

In recent years, research has illuminated a link between active galactic nuclei (AGNs) and the evolution of their host galaxies. Studies have found that a galaxy's central black hole mass is correlated with the mass of the central bulge (e.g., Ferrarese \& Merritt 2000; Gebhardt et al. 2000; Tremaine et al. 2002; Shields et al. 2003; Marconi \& Hunt 2003; Häring \& Rix 2004). In the local universe, high-luminosity AGNs are preferentially found in early-type galaxies with young mean stellar ages (Kauffmann et al. 2003; Heckman et al. 2004). As the redshift increases, the cosmic rates of both star formation and AGN activity increase (Boyle \& Terlevich 1998; Bluck et al. 2011), and, at high redshift, intensely star-forming submillimeter galaxies have been found to have an AGN fraction of $\sim 20 \%-30 \%$ (Laird et al. 2010; Georgantopoulos et al. 2011).

The connection between AGNs and host evolution is also observed in optical colors. Studies have shown that galaxies are organized based on rest-frame color, separating into a "red sequence" and a "blue cloud" on a color-magnitude diagram (CMD; e.g., Strateva et al. 2001; Baldry et al. 2004; Weiner et al. 2005). The sparsely populated "green valley" between these regions is thought to be a transitional area, where blue cloud galaxies are rapidly migrating onto the red sequence after the cessation of star formation (Faber et al. 2007). A proposed mechanism for this quick transition is AGN feedback, where the AGN activity, driven by major mergers or tidal interactions, causes a truncation of star formation and leads to the color evolution onto the red sequence (Hopkins et al. 2005, 2007; Springel et al. 2005; Somerville et al. 2008). This theory is supported by a number of studies which find an association between AGN activity and the green valley (Nandra et al. 2007; Georgakakis et al. 2008; Silverman et al. 2008; Kocevski et al. 2009b; Hickox et al. 2009; Schawinski et al. 2009). However, some studies using mass-selected samples have found a more uniform color distribution of AGN hosts (Silverman et al. 2009b; Xue et al. 2010). In addition, Cardamone et al. (2010) have found that $\sim 75 \%$ of the green-valley AGN hosts are dust-reddened members of the blue cloud, although these results are apparently in conflict with the more recent studies of Rosario et al. (2011). Studies examining AGNs for recent merger activity have also found mixed results (e.g., Sánchez et al. 2005; Pierce et al. 2007; Georgakakis et al. 2008; Kocevski et al. 2009a, 2011b).

Menanteau et al. (2001) offer an alternative to major mergerdriven AGNs, where AGNs are instead triggered by minor mergers, mainly in red galaxies. Star formation in this scenario would only be briefly re-ignited at the cores of many of these systems and the galaxies would return to the red sequence after its cessation. These AGNs would then represent a red-sequence population evolving in luminosity space rather than blue cloud 
Table 1

Properties of Observed ORELSE Structures

\begin{tabular}{|c|c|c|c|c|c|c|c|c|c|}
\hline Structure & $\begin{array}{l}\text { R.A. }^{\mathrm{a}} \\
(\mathrm{J} 2000)\end{array}$ & $\begin{array}{l}\text { Decl. }^{\mathrm{a}} \\
(\mathrm{J} 2000)\end{array}$ & $\langle z\rangle$ & $\begin{array}{c}z \text { Lower } \\
\text { Bound }\end{array}$ & $\begin{array}{l}z \text { Upper } \\
\text { Bound }\end{array}$ & $\begin{array}{c}\text { Number of } \\
\text { Clusters/Groups }\end{array}$ & $\begin{array}{c}\sigma \\
\text { Range }^{b}\end{array}$ & $\begin{array}{l}\text { Confirmed } \\
\text { Members }^{c}\end{array}$ & $\begin{array}{l}\text { Confirmed } \\
\text { AGNs }^{c}\end{array}$ \\
\hline $\mathrm{Cl1604}$ & 160415 & +431624 & 0.90 & 0.84 & 0.96 & 10 & $300-800$ & 531 & 10 \\
\hline $\mathrm{Cl} 10023$ & 002351 & +042255 & 0.84 & 0.82 & 0.87 & 4 & $200-500$ & 244 & 7 \\
\hline $\mathrm{Cl} 1324$ & 132445 & +303418 & 0.76 & 0.65 & 0.79 & 10 & 200-900 & 393 & 6 \\
\hline RXJ1821 & 182132.4 & +682756 & 0.82 & 0.80 & 0.84 & 1 & $910 \pm 80$ & 90 & 3 \\
\hline RXJ1757 & 175719.4 & +663129 & 0.69 & 0.68 & 0.71 & 1 & $650 \pm 120$ & 42 & 1 \\
\hline
\end{tabular}

Notes.

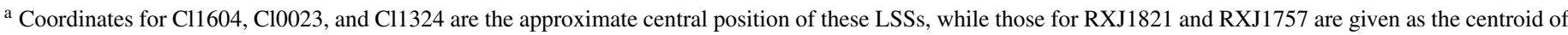
the peak of diffuse X-ray emission associated with the respective cluster.

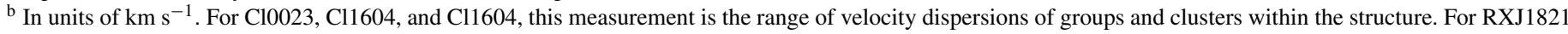
and RXJ1757, it is the dispersion of the single cluster. All velocity dispersions are measured within $1 \mathrm{Mpc}$.

${ }^{c}$ Spectroscopically confirmed objects $(Q=3,4)$ within the redshift bounds of the structure; see Section 3.1 for quality flag details.

galaxies migrating onto the red sequence. In support of this theory, several studies have found AGN activity associated with blue early-type galaxies or red galaxies with blue cores (Lee et al. 2006; Martel et al. 2007; Kocevski et al. 2009b). Additionally, Ciotti \& Ostriker (2007) propose that recycled stellar material in elliptical galaxies could fuel central starbursts and black hole accretion. Without precise observations of morphologies, the effects of these two models would appear similar.

Other plausible models exist as well, some important only in certain regimes. For example, at high redshift $(z \gtrsim 2)$, Bournaud et al. (2011) propose that violent disk instabilities could lead to powerful nuclear activity, while Hopkins \& Hernquist (2006) present a model where lower luminosity AGNs could be triggered by mild disk instabilities or gas funneled through bars. Note that the properties of individual AGNs are widely varying, and it could be the case that any number of modes contribute to AGN triggering, with different contributions in different mass and/or redshift regimes.

To better understand how AGNs are triggered and the role they play in galaxy evolution, it is useful to study large-scale structures (LSSs) at high redshift. These environments contain a large number of galaxies in the process of transitioning from actively star forming to passive, and there is evidence that nuclear activity increases at higher redshifts (Boyle \& Terlevich 1998; Eastman et al. 2007; Kartaltepe et al. 2007; Bluck et al. 2011).

To this end, we have examined the X-ray-selected AGN population within five LSSs at redshifts of $z \approx 0.7-0.9$. These structures are in varying states of evolution and include complex superclusters, an interacting supergroup, and isolated clusters. Each has been studied extensively by the Observations of Redshift Evolution in Large-Scale Environments (ORELSE) survey, which is searching for LSSs in the vicinities of 20 known clusters between $z=0.6$ and 1.3. The survey has compiled extensive multi-wavelength data sets for each structure, which include multi-band optical, radio, and X-ray imaging, as well as thousands of spectroscopic redshifts (Lubin et al. 2009).

In this paper, we present analyses of X-ray point sources and the properties of the AGN population within the following five LSSs: the C11604 and Cl1324 superclusters at $z \approx 0.9$ and $z \approx 0.76$, respectively, the $\mathrm{Cl} 0023+0423$ supergroup at $z \approx 0.82$, and two X-ray selected and relatively relaxed, isolated clusters, RXJ1757.3+6631 at $z=0.69$ and RXJ1821.6+6827 at $z=0.84$. We study this sample with a combination of Chandra X-ray data, optical imaging, and near-IR and optical spectroscopy. For our cosmological model, we assume $\Omega_{m}=$ $0.3, \Omega_{\Lambda}=0.7$, and $h_{70}=H_{0} / 70 \mathrm{~km} \mathrm{~s}^{-1} \mathrm{Mpc}^{-1}$.

We discuss the clusters and superclusters in our survey in Section 2. Observations, data reduction, and techniques are discussed in Section 3. The global properties of our sample are discussed in Section 4. The statistical measurements of cumulative source counts are covered in Section 5. Analysis of the AGNs is presented in Section 6.

\section{THE ORELSE STRUCTURE SAMPLE}

In this section we describe the five structures in our sample, which are succinctly summarized in Table 1 . The redshift boundaries used in the following analyses were determined by visually examining each structure's redshift histogram. Delineating where structures end is not straightforward, with some having associated filaments or possible nearby sheets. The redshift boundaries are chosen with the aim to include all galaxies which could be part of each overall LSS.

\subsection{The Cl1604 Supercluster}

The $\mathrm{Cl1604}$ supercluster at $z \approx 0.9$ is one of the largest structures studied at high redshift. It consists of at least 10 clusters and groups and spans $100 h_{70}^{-1} \mathrm{Mpc}$ along the line of sight and $13 h_{70}^{-1} \mathrm{Mpc}$ in the plane of the sky (Lubin et al. 2000; Gal \& Lubin 2004; Gal et al. 2008; Lemaux et al. 2009). The massive member clusters Cl1604+4304 and C11604+4321 were first discovered in the optical survey of Gunn et al. (1986). The proximity of the clusters suggested that they were components of a larger structure. Further wide field imaging has revealed 10 distinct red galaxy overdensities, suggesting the existence of a supercluster (Lubin et al. 2000; Gal \& Lubin 2004; Gal et al. 2008). Spectroscopic observations have confirmed four of the overdensities to be clusters with velocity dispersions in excess of $500 \mathrm{~km} \mathrm{~s}^{-1}$, while four others were confirmed to be poor clusters or groups with dispersions in the range $300-500 \mathrm{~km} \mathrm{~s}^{-1}$ (Postman et al. 1998, 2001; Gal et al. 2005, 2008).

The two most massive clusters in $\mathrm{Cl1604}$ have associated diffuse X-ray emission. Cl1604+4304 and Cl1604+4314, hereafter Clusters A and B, have measured bolometric X-ray luminosities of $15.76 \pm 1.48$ and $11.64 \pm 1.49 \times 10^{43} h_{70}^{-2} \mathrm{erg} \mathrm{s}^{-1}$ and X-ray temperatures of $3.50_{-1.08}^{+1.82}$ and $1.64_{-0.45}^{+0.65} \mathrm{keV}$, respectively (Kocevski et al. 2009a). While these values place Cluster A on the $\sigma-T$ curve for virialized clusters, Cluster B is well off from it, suggesting that Cluster A is relaxed while Cluster B is not. 
All other clusters have only an upper limit on their bolometric luminosity of $7.4 \times 10^{43} h_{70}^{-2} \mathrm{erg} \mathrm{s}^{-1}$ (Kocevski et al. 2009a).

While many galaxies in $\mathrm{Cl} 1604$ have substantial [O II] emission, near-infrared spectroscopy has shown that a significant portion of this emission is due to contributions from lowionization nuclear emission-line regions (LINERs) and Seyferts (Lemaux et al. 2010). Also, Kocevski et al. (2011a) studied $24 \mu \mathrm{m}$ selected galaxies in and around three clusters and three groups in $\mathrm{Cl} 1604$ using the Multiband Imaging Photometer for Spitzer (MIPS; Rieke et al. 2004) and found evidence for recent starburst activity and an infalling population. Analysis of the morphologies using the Advanced Camera for Surveys (ACS; Ford et al. 2003) on the Hubble Space Telescope (HST) revealed that many of these $24 \mu \mathrm{m}$ bright galaxies were disturbed, indicating mergers and interactions were likely responsible for starburst activity.

We refer the reader to Kocevski et al. (2009a), Gal et al. (2008), and Lemaux et al. (2011) for more details on the data processing, supercluster properties, and observations.

\section{2. $\mathrm{ClOO23+0423}$}

The $\mathrm{Cl} 0023+0423$ structure at $z \approx 0.84$, hereafter $\mathrm{Cl0023}$, was also discovered as an overdensity in the optical survey of Gunn et al. (1986). The structure was later observed by Oke et al. (1998) using the Low-Resolution Imaging Spectrograph (LRIS; Oke et al. 1995) on the Keck 10 m telescope, where the overdensity was resolved into two structures. Further study has shown that the structure consists of four merging galaxy groups separated by approximately $3000 \mathrm{~km} \mathrm{~s}^{-1}$ in radial velocity and $\sim 0.25 \mathrm{Mpc}$ on the plane of the sky (Lubin et al. 1998, 2009). The constituent groups have measured velocity dispersions within $1.0 h_{70}^{-1} \mathrm{Mpc}$ of $480 \pm 170,430 \pm 70,290 \pm 80$, and $210 \pm 30 \mathrm{~km} \mathrm{~s}^{-1}$ (Lubin et al. 2009). Simulations suggest that the groups will merge to form a cluster of mass $\sim 5 \times 10^{14} M_{\odot}$ within $\sim 1$ Gyr (Lubin et al. 1998).

Lubin et al. (2009) found C10023 to have a large blue population, with $51 \%$ of the galaxies bluer than their red galaxy color-color cut, down to an $i^{\prime}$-band magnitude of 24.5. Spectroscopic analysis found that $\sim 80 \%$ of the galaxies had measurable [O II] emission, which, because of the large blue population, is most likely due to ongoing star formation. 5

We refer the reader to Lubin et al. (2009) and Kocevski et al. (2009c) for more details on the supergroup properties and observations.

\subsection{The Cl1324 Supercluster}

The $\mathrm{Cl1324}$ supercluster is an LSS at $z \approx 0.76$. The two most massive clusters in the structure, Cl1324+3011 at $z=0.76$

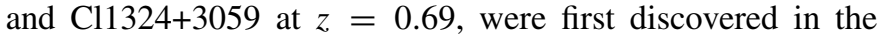
optical survey of Gunn et al. (1986). Because of the proximity of the clusters on the sky and in redshift space, this structure was chosen for the ORELSE survey to investigate the possible existence of structure in the field. Wide-field imaging has revealed a total of ten clusters and groups, detected through red galaxy overdensities, and, despite extensive spectroscopy, only four have been spectroscopically confirmed as constituent clusters or groups (see R. R. Gal et al. 2012, in preparation).

Cl1324+3011 was previously studied in Lubin et al. (2002, 2004), where a velocity dispersion of $1016_{-93}^{+126} \mathrm{~km} \mathrm{~s}^{-1}$ and a

\footnotetext{
5 Refer to Section 4 for a discussion of [O II] emission.
}

temperature of $2.88_{-0.49}^{+0.71} \mathrm{keV}$, using XMM-Newton, were measured for the cluster. According to these measurements, the cluster does not fall close to the $\sigma-T$ curve for virialized clusters, which would imply that it is not well relaxed. New Chandra results for $\mathrm{Cl1324}$ are presented in N. Rumbaugh et al. (2012, in preparation), including new X-ray temperatures for Cl1324+3011 and Cl1324+3059. In addition, we present here updated velocity dispersions for these two clusters of $930 \pm 120$ and $870 \pm 120 \mathrm{~km} \mathrm{~s}^{-1}$, respectively. The new measurements

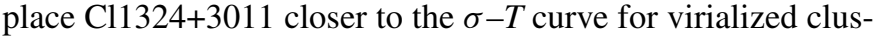
ters, only offset by $\sim 1 \sigma$. While the older measurements suggested the cluster was not relaxed, the new measurements are more consistent with virialization. Similar to C11324+3011, Cl1324+3059 is offset from the curve, but still by less than $1 \sigma$.

The photometric and spectroscopic observations of the Cl1324 supercluster will be covered in full in R. R. Gal et al. (2012, in preparation). In this paper, we present redshift histograms of the full structure and velocity dispersions for the four confirmed groups and clusters, as well as the Chandra observations.

\section{4. $R X J 1821.6+6827$}

The X-ray-selected cluster RXJ1821.6+6827, hereafter RXJ1821, at $z=0.82$, was the highest redshift cluster discovered in the ROSAT North Ecliptic Pole (NEP) survey, where it is also referred to as NEP5281 (Gioia et al. 2003; Henry et al. 2006). Using XMM-Newton data, the cluster was found to have slightly elongated diffuse $X$-ray emission with a measured bolometric luminosity of $1.17_{-0.18}^{+0.13} \times h_{70}^{-2}$ erg s$^{-1}$ and a temperature of $4.7_{-0.7}^{+1.2} \mathrm{keV}$ (Gioia et al. 2004). The same study measured a velocity dispersion of $775_{-113}^{+182} \mathrm{~km} \mathrm{~s}^{-1}$ using 20 cluster members. Later analysis by Lubin et al. (2009) used 40 galaxies within $1 \mathrm{Mpc}$ to measure a velocity dispersion of $926 \pm 77 \mathrm{~km} \mathrm{~s}^{-1}$. Redshift histograms of RXJ1821 are characteristic of a single, isolated structure, although a small kinematically associated group has been detected to the south (Lubin et al. 2009). While the temperature and dispersion measurements place the cluster near the $\sigma-T$ relation for virialized clusters, the elongated X-ray emission could be indicative of still ongoing formation of the cluster.

Lubin et al. (2009) measured a blue fraction of only $24 \%$ for RXJ1821, down to a magnitude limit of $i^{\prime}=24.5$. They found a population dominated by massive, old (formation epoch of $z_{f} \sim 2-3$ ) galaxies, along with fainter galaxies that were quenched more recently. They also found that $36 \%$ of the galaxies had detectable [O II] emission. Near-IR spectroscopy of a subset of these [O II] emitting galaxies suggests that some of the emission is due to LINER or AGN activity (Lemaux et al. 2010).

We refer the reader to Lubin et al. (2009) for more details on the data processing, cluster properties, and observations.

\section{5. $R X J 1757.3+6631$}

The $z=0.69$ cluster RXJ1757.3+6631, hereafter RXJ1757, was discovered as part of the ROSAT NEP survey, where it is also identified as NEP200 (Gioia et al. 2003). Gioia et al. (2003) found the structure to have an X-ray luminosity of $8.6 \times 10^{43} h_{70}^{-2} \mathrm{erg} \mathrm{s}^{-1}$ in the $0.5-2.0 \mathrm{keV}$ band. The structure is dominated by a single, large cluster. In this paper, we present a velocity dispersion, redshift histograms, and analysis of X-ray point sources for RXJ1757, none of which have been previously published. 
Table 2

Spectroscopic Observation Characteristics

\begin{tabular}{lcc}
\hline \hline Structure & $\begin{array}{c}\text { Central } \\
\lambda(\AA)\end{array}$ & $\begin{array}{c}\text { Approx. Spectral } \\
\text { Coverage }(\AA)\end{array}$ \\
\hline C10023 & $7500-7850$ & $6200-9150$ \\
C11604 & 7700 & $6385-9015$ \\
C11324 & 7200 & $5900-8500$ \\
RXJ1821 & $7500-7800$ & $6200-9100$ \\
RXJ1757 & $7000-7100$ & $5700-8400$ \\
\hline
\end{tabular}

\section{OBSERVATIONS AND REDUCTION}

\subsection{Optical and NIR Observations}

Ground-based optical imaging data were obtained with the Large Format Camera (LFC; Simcoe et al. 2000) on the Palomar $5 \mathrm{~m}$ telescope. Observations were taken using the Sloan Digital Sky Survey $r^{\prime}, i^{\prime}$, and $z^{\prime}$ filters. The $5 \sigma$ point source limiting magnitudes for the five fields ranged from 25.5-25.1, 25.0-24.5, and 23.6-23.3 in the $r^{\prime}, i^{\prime}$, and $z^{\prime}$ bands, respectively.

Cl1604 was also imaged using ACS. The HST imaging for Cl1604 consists of 17 ACS pointings designed to image 9 of the 10 galaxy density peaks in the field. Observations were taken using the F606W and F814W bands. These bands roughly correspond to broadband $V$ and $I$, respectively.

Our photometric catalog is complemented by an unprecedented amount of spectroscopic data. For this part of the study, we used the Deep Imaging Multi-Object Spectrograph (DEIMOS; Faber et al. 2003) on the Keck II 10 m telescope. In addition, Cl1604 and RXJ1821 have some LRIS coverage (see Oke et al. 1998; Gal \& Lubin 2004; Gioia et al. 2004). DEIMOS has a wide field of view $(16.9 \times 5$ '.0), high efficiency, and is able to position over 120 targets per slit mask, which makes the instrument ideal for establishing an extensive spectroscopic catalog. We targeted objects down to an $i^{\prime}$-band magnitude of 24.5. On DEIMOS, we used the 1200 line $\mathrm{mm}^{-1}$ grating, blazed at $7500 \AA$, and $1^{\prime \prime}$ slits. These specifications create a pixel scale of $0.33 \AA$ pixel $^{-1}$ and an FWHM resolution of $\sim 1.7 \AA$, or $68 \mathrm{~km} \mathrm{~s}^{-1}$. The central wavelength was varied from structure to structure and sometimes between different masks for the same field. Central wavelengths for the spectroscopic observations for the five fields and the approximate spectral coverages are displayed in Table 2. When more than one central wavelength was used per field, a range is given. Total exposure times for the observations are in the range of 1-4 hr per mask.

Spectroscopic targets were chosen based on color and magnitude. The number of spectroscopic targets in each field is shown in Table 3. Redshifts were determined or measured for all targets and given a quality flag value, $Q$, where $Q=1$ indicates that we could not determine a secure redshift, $Q=2$ means a redshift was obtained using features that were only marginally detected, $Q=3$ means one secure and one marginal feature were used to calculate the redshift, and $Q=4$ means at least two secure features were used. Those sources determined to be stars were given a flag of -1 . See Gal et al. (2008) for more details on quality flags and the spectral targeting method. For our analysis, redshifts with $Q=-1,3,4$ were deemed satisfactory, and the number of such sources in each field is shown in Table 3.

Spectroscopic data have been previously presented for the Cl1604 supercluster, Cl0023, and RXJ1821 as part of the ORELSE survey. We present new data for each of these structures, as well as for the Cl1324 supercluster and RXJ1757. We include ten DEIMOS masks for C11324, with exposure times ranging from $6635 \mathrm{~s}$ to $10,800 \mathrm{~s}$, and four DEIMOS masks for RXJ1757, with exposure times ranging from $7200 \mathrm{~s}$ to $14,730 \mathrm{~s}$. We include a total of 18 spectroscopic masks for Cl1604, 6 more than what were included in Gal et al. (2008). ${ }^{6}$ We include nine total masks for $\mathrm{Cl0023}$, four more than in Kocevski et al. (2009c). We include three masks for RXJ1821, one more than in Lubin et al. (2009). Many of the new targets were optical counterparts to X-ray sources. From our measured redshifts with $Q=3$ or 4 from the new masks, we find 114 new galaxies in Cl1604 within $0.84<z<0.96,104$ new galaxies in $\mathrm{Cl} 10023$ within $0.82<z<0.87,{ }^{7}$ and 5 new galaxies in RXJ1821 within $0.80<z<0.84$.

\subsection{X-Ray Observations}

All X-ray imaging of the clusters was conducted with the Advanced CCD Imaging Spectrometer (ACIS) of the Chandra $X$-ray Observatory, using the ACIS-I array (PI: L. M. Lubin). ${ }^{8}$ This array has a $16.9 \times 16.9$ field of view. Some of the five structures were imaged with one pointing and some with two, but every pointing had the same approximate total exposure time of $50 \mathrm{ks}$. Cl0023, RXJ1821, and RXJ1757 were each imaged with one pointing of the array. Cl1604 and Cl1324,

\footnotetext{
6 See Lemaux et al. (2011) for a detailed description of all Cl1604 spectroscopic observations and results.

7 Note that while we define the bounds of $\mathrm{Cl} 10023$ as $0.82<z<0.87$ here, Kocevski et al. (2009c) use $0.820<z<0.856$, excluding a sheet of galaxies at $z \approx 0.86-0.87$. In the smaller range of $0.820<z<0.856$, we add 96 new galaxies. We use a wider redshift range in order to be consistently liberal in our structure boundaries.

8 Details of the Chandra observations, such as ID numbers, are described in N. Rumbaugh et al. (2012, in preparation).
}

Table 3

Number of Spectroscopic Targets, X-Ray Sources, and Member Galaxies

\begin{tabular}{|c|c|c|c|c|c|c|}
\hline Structure & $\begin{array}{c}\text { Spectroscopic } \\
\text { Targets }\end{array}$ & $\begin{array}{l}\text { Spectroscopic } \\
\text { Redshifts }^{\mathrm{a}}\end{array}$ & $\begin{array}{l}\text { X-Ray Sources, } \\
\quad>3 \sigma(>2 \sigma)^{\mathrm{b}}\end{array}$ & $\begin{array}{c}\text { X-Ray Sources, } \\
\text { Matched }^{\mathrm{c}}\end{array}$ & $\begin{array}{l}\text { Attempted } \\
\text { Redshifts }^{\mathrm{d}}\end{array}$ & $\begin{array}{l}\text { Confirmed } \\
\text { Redshifts }^{\mathrm{a}}\end{array}$ \\
\hline C11604 & 2465 & 1785 & $158(213)$ & $112(128)$ & $43(48)$ & $38(42)$ \\
\hline $\mathrm{Cl} 0023$ & 1136 & 892 & $94(133)$ & $58(72)$ & $39(49)$ & $26(32)$ \\
\hline Cl1324 & 1419 & 1155 & $174(217)$ & $126(133)$ & $38(40)$ & $28(30)$ \\
\hline RXJ1821 & 351 & 306 & $102(132)$ & $64(72)$ & $15(18)$ & $10(13)$ \\
\hline RXJ1757 & 549 & 421 & $87(107)$ & $57(62)$ & 18(19) & $9(9)$ \\
\hline
\end{tabular}

\footnotetext{
Notes.

${ }^{a}$ Only includes redshifts with quality flag $Q=-1$, 3, or 4; see Section 3.1 for flag details.

${ }^{b}$ Includes sources with a significance $>3 \sigma(>2 \sigma)$ in at least one of the three bands: soft, hard, or full.

c X-ray sources matched to optical counterparts.

d Includes all X-ray sources that were targeted for spectroscopy, regardless of the quality of measured redshift.
} 
with angular sizes in excess of $20^{\prime}$, were observed with two pointings each. For Cl1604, the two pointings are meant to cover as much of the structure as possible, and there is a small overlap $\left(\sim 30 \operatorname{arcmin}^{2}\right)$. For Cl1324, the two pointings are centered near the two largest and originally discovered clusters C11324+3011 and Cl1324+3059. There is an approximately $13^{\prime}$ gap between the north and south pointings.

In this paper, we present new Chandra data for Cl1324, RXJ1821, and RXJ1757.

\subsection{X-Ray Data Reduction and Photometry}

The reduction of the data was conducted using the Chandra Interactive Analysis of Observations 4.2 software (CIAO; Fruscione et al. 2006). Each observation was filtered by energy into three bands: $0.5-2 \mathrm{keV}$ (soft), 2-8 keV (hard), and 0.5-8 keV (full). Data were checked for flares using dmextract and the Chandra Imaging and Plotting System (ChIPS) routine $l c \_$clean. Exposure maps were created using the routine merge_all. For vignetting correction, exposure maps were normalized to their maximum value, then images were divided by this normalized exposure map.

To locate point sources, the routine wavdetect was run on each observation, without vignetting correction, using wavelet scales of $2^{i / 2}$ pixels, with $i$ ranging from 0 to 8 . A threshold significance of $10^{-6}$ was used, which would imply fewer than one spurious detection per ACIS chip, which has dimensions of $1024 \times 1024$ pixels (0'.492 per pixel). However, this assumes a uniform background, which is almost certainly not the case. To measure realistic detection significances, we instead used photometric results explained below. Point source detection was carried out on images in each of the soft, hard, and full bands separately. For C11604 and Cl1324, wavdetect was run on each of the two pointings separately. Output object positions from the three different bands were cross-correlated to create one final composite list for each field.

We carried out follow-up photometry on the point sources. Circular apertures containing 95\% of the flux were created for each point source using the point-spread function (PSF) libraries in the Chandra calibration database. For Chandra, the PSF depends on both energy and off-axis angle. For the soft and hard bands, respectively, we used the PSF libraries for energies of 1.497 and $4.510 \mathrm{keV}$. Photometry was carried out on the vignetting-corrected images in the soft and hard bands. Background counts for each source were calculated in annuli with inner and outer radii of $1.2 \times R_{95}$ and $2.4 \times R_{95}$, where $R_{95}$ is the radius of the circular aperture containing $95 \%$ of the flux. Since only $95 \%$ of the flux is enclosed, net counts calculated from the apertures were multiplied by $1 / 0.95$ to recover all the counts. Full band counts were calculated by summing those from the soft and hard bands. The results of the photometry were used to calculate detection significances for the sources in each of the three bands using

$$
\sigma=C /(1.0+\sqrt{0.75+B})
$$

where $C$ is the net photon counts from the source and $B$ is the background counts (Gehrels 1986). X-ray sources with significances $<2 \sigma$ were rejected as spurious. With $\sim 75 \%$ of accepted sources having detection significances $>3 \sigma$, and the remaining $\sim 25 \%$ with detection significances between $2 \sigma$ and $3 \sigma$, we expect a spurious detection rate of $\lesssim 1.5 \%$, based on a normal distribution.

Due to the low number of photons observed for many sources, we opted to normalize a power-law spectral model
Table 4

Count Rate to Flux Conversion Factors and H I Column Densities

\begin{tabular}{lccc}
\hline \hline Structure & $\begin{array}{c}N_{\mathrm{HI}_{\mathrm{I}}}{ }^{\mathrm{a}} \\
\left(10^{20} \mathrm{~cm}^{-2}\right)\end{array}$ & $\begin{array}{c}\text { Count Rate to } \\
\text { Flux Conversion, } \\
\text { Soft Band }\end{array}$ & $\begin{array}{c}\text { Count Rate to } \\
\text { Flux Conversion, } \\
\text { Hard Band }\end{array}$ \\
\hline Cl1604 & 1.2 & 6.12 & 22.2 \\
C10023 & 2.7 & 6.37 & 22.3 \\
Cl1324 & 1.1 & 6.16 & 22.2 \\
RXJ1821 & 5.6 & 8.23 & 22.3 \\
RXJ1757 & 4.0 & 6.60 & 22.2 \\
\hline
\end{tabular}

Notes.

a Galactic neutral hydrogen column density, using the data set of Dickey \& Lockman (1990).

b X-ray net count rate to unabsorbed flux conversion factor, in units of $10^{-12} \mathrm{erg} \mathrm{cm}^{-2}$.

to the net count rate of individual point sources to determine fluxes. We assumed a photon index of $\gamma=1.4$, which is the approximate slope of the X-ray background in the hard band (Tozzi et al. 2001; Kushino et al. 2002). Count rates were calculated by dividing net counts by the nominal exposure time at the aim point of the appropriate observation. The galactic neutral hydrogen column density was calculated at the aim point of each observation using the Colden tool from the Chandra proposal toolkit, which uses the data set of Dickey \& Lockman (1990). Hydrogen column densities and derived net count rate to unabsorbed flux conversion factors for each field are summarized in Table 4. Conversion factors were determined separately for the different pointings of Cl1604 and Cl1324, but did not differ to the three significant figures listed in the table.

\subsection{Optical Matching}

To search for AGNs within the individual clusters, we matched X-ray and optical sources. In order to increase our completeness, the input to the matching included all point sources detected by wavdetect, regardless of significance in any of the three bands. We used the maximum likelihood ratio technique described in Kocevski et al. (2009a), which was developed by Sutherland \& Saunders (1992) and also used by Taylor et al. (2005) and Gilmour et al. (2007). Our technique is similar to Kocevski et al. (2009a), but with a few key differences. The main statistic calculated in each case is the likelihood ratio (LR), which estimates the probability that a given optical source is the genuine match to a given point source relative to the arrangement of the two sources arising by chance. The LR is given by the equation

$$
\mathrm{LR}_{i, j}=\frac{w_{i} \exp \left(-r_{i, j}^{2} / 2 \sigma_{j}^{2}\right)}{\sigma_{j}^{2}} .
$$

Here, $r_{i, j}$ is the separation between objects $i$ and $j, \sigma_{j}$ is the positional error of object $j,{ }^{9}$ and $w_{i}=1 / n\left(<m_{i}\right)$ is the inverse of the number density of optical sources with magnitude brighter than $m_{i}$. The inclusion of the latter quantity is designed to weight against matching to fainter optical objects. However, in our analysis, we found that this particular weighting, used by Kocevski et al. (2009a), of $1 / n\left(<m_{i}\right)$ gave too much favor to bright objects, even when they were much farther from an

\footnotetext{
9 Positional errors of X-ray sources were calculated using the method of Kim et al. (2007). Optical positional errors were small compared to those of X-ray objects, and were considered negligible.
} 
$\mathrm{X}$-ray object than a faint source. We adjusted the weighting to $w_{i}=n\left(<m_{i}\right)^{-1 / 2}$. We found that this change did not have a large overall effect but changed some borderline cases where a bright object with a large separation from the point had been chosen over a dimmer, much closer object. This includes one case, which prompted the adjustment, where spectroscopy showed that an M-type star was matched instead of a probable AGN, even though the $\mathrm{M}$ star was three times farther from the X-ray source.

For each field, except C11604, $n\left(<m_{i}\right)$ was measured using $i^{\prime}$ magnitudes from our LFC catalogs. For C11604, ACS data were also available, but these observations did not cover the entire field. All objects were matched to the LFC catalogs. When possible, objects were also matched using the F814W magnitude from the ACS catalogs and matches to ACS objects took precedence over matches to LFC objects.

From the LR, we use Monte Carlo simulations to derive the probability that a given match is genuine. We ran 10,000 trials for each X-ray object. In each trial, the object's position was randomized and the LR was calculated based on nearby optical sources. The LR for a given X-ray object to optical counterpart pairing, $\mathrm{LR}_{i, j}$, was compared against the distribution of LRs from the 10,000 Monte Carlo trials. We calculated the reliability as

$$
R_{i, j}=1-\frac{N\left(\mathrm{LR}_{j}>\mathrm{LR}_{i, j}\right)}{10,000},
$$

where $N\left(\mathrm{LR}_{j}>\mathrm{LR}_{i, j}\right)$ is the number of matches, to any optical source, across all 10,000 trials for that X-ray object with LR greater than $\mathrm{LR}_{i, j} . R_{i, j}$ can be interpreted as the probability that optical source $i$ is the true match of X-ray source $j$, in the case of only one optical candidate. When there are multiple candidates, we used the method of Rutledge et al. (2000) to calculate the probability that optical source $i$ is the true match of X-ray source

$$
P_{i, j}=\frac{R_{i, j} \prod_{k \neq i}^{N}\left(1-R_{k, j}\right)}{S} .
$$

The probability that no optical source is the true match is

$$
P_{\text {none }, j}=\frac{\prod_{k=1}^{N}\left(1-R_{k, j}\right)}{S},
$$

where $N$ is the total number of optical candidates and $S$ is a normalization factor defined so that $\sum_{i=1}^{N}\left(P_{i, j}\right)+P_{\text {none, } j}=1$.

For an X-ray source with a single optical counterpart, a match was considered genuine if $P_{\text {none, } j}<0.15$. For X-ray sources with multiple optical counterparts, a genuine match was chosen if $\sum_{i} P_{i, j}>0.85$ (which is equivalent to $P_{\text {none, } j}<0.15$ ) and $P_{i, j}>4 \sum_{k \neq i} P_{k, j}$ for any one object $i$. If the first condition was true, but the second was not, all objects with $P_{i, j}>0.2$ were considered as matches. In subsequent sections, only one optical counterpart was considered for each X-ray source. In almost all cases, the highest probability match was used. However, in several cases, spectra of the primary and secondary matches indicated that the secondary match was an AGN, and thus more likely to be a genuine match. Note that our threshold is a deviation from Kocevski et al. (2009a). They used $P_{\text {none, } j}<0.2$ instead of 0.15 . We decided to use the more stringent threshold of 0.15 , which has been used by others (Mann et al. 2002; Taylor et al. 2005), to better limit the number of false matches. The more restrictive cutoff omitted $\lesssim 10$ sources per field. We determined this threshold through visual inspection of potential matches. This calibration entailed determining at what approximate level of $P_{\text {none, } j}$ most matches visually seemed spurious. However, optical candidates above our threshold were also visually scrutinized ( $\lesssim 3 \%$ of the total), and some were accepted after this inspection where we felt the matching algorithm had failed. Note that setting a threshold for genuine matches is not entirely objective, and a precedent has been set for accomplishing this with visual inspection (e.g., Mann et al. 1997). In Table 3, we list, for each field, the number of X-ray sources detected at $>3 \sigma(>2 \sigma)$ in one of the three bands, as well as the number of those sources matched to optical counterparts.

\section{GLOBAL PROPERTIES OF THE ORELSE STRUCTURES}

The five structures in our sample span a range of evolutionary states. They include C10023, whose four constituent groups are still in the process of merging to form a single cluster; the two isolated X-ray-selected clusters, RXJ1757 and RXJ1821, which appear to be in a more evolved and relaxed state; and the two superclusters, Cl1604 and C11324. We would like to compare the AGNs within our sample based on the evolutionary states of the structures to which they belong, which could shed light on how the AGNs in these systems are being triggered. In order to make such a comparison, we first present the global properties of the five ORELSE structures.

\subsection{Redshift Distributions}

The $\mathrm{Cl1604}$ supercluster, Cl0023, and RXJ1821 have all been studied previously as part of the ORELSE survey (see Section 2 for individual references), although we have gathered new data on each, as described in Section 3.1. While individual clusters in C11324 have been studied, the properties of the supercluster as a whole have not. In this section we do a preliminary exploration of this structure, which will be covered more thoroughly in an upcoming paper (R. R. Gal et al. 2012, in preparation). The cluster RXJ1757 was studied only as a part of the ROSAT NEP survey (Gioia et al. 2003), in little detail. Here, we present new redshift histograms of these last two structures derived from our ORELSE data.

\subsection{1. $C l 1324$}

Figure 1(a) shows all confirmed redshifts in the spatial vicinity of Cl1324. We can see two peaks in the histogram, at $z \approx 0.695$ and $z \approx 0.755$. These peaks coincide with the two largest clusters in the structure, C11324+3011 and C11324+3059. From the distribution of red galaxies, we find ten overdensities in the supercluster, some of which can be observed in the redshift histogram. So far, we have confirmed four clusters and groups to be constituents, shown in Table 5, with coordinates, redshifts, and measured velocity dispersions given. Additional multiobject spectroscopy to confirm the nature of the other red galaxy overdensities is planned.

\subsection{2. $R X J 1757$}

The redshift histograms for RXJ1757 are displayed in Figures $1(\mathrm{c})$ and (d). At $z \approx 0.69$ and $z \approx 0.9$, we see two peaks in the distribution. The former is the overdensity associated with RXJ1757. When we examine the spatial distribution of the higher redshift peak, we find that the galaxies in its vicinity are distributed nearly uniformly across the field of view, implying a sheet of galaxies.

Looking at the redshift distribution of confirmed galaxies within the bounds of RXJ1757 (Figure 1(d)), we can see the 

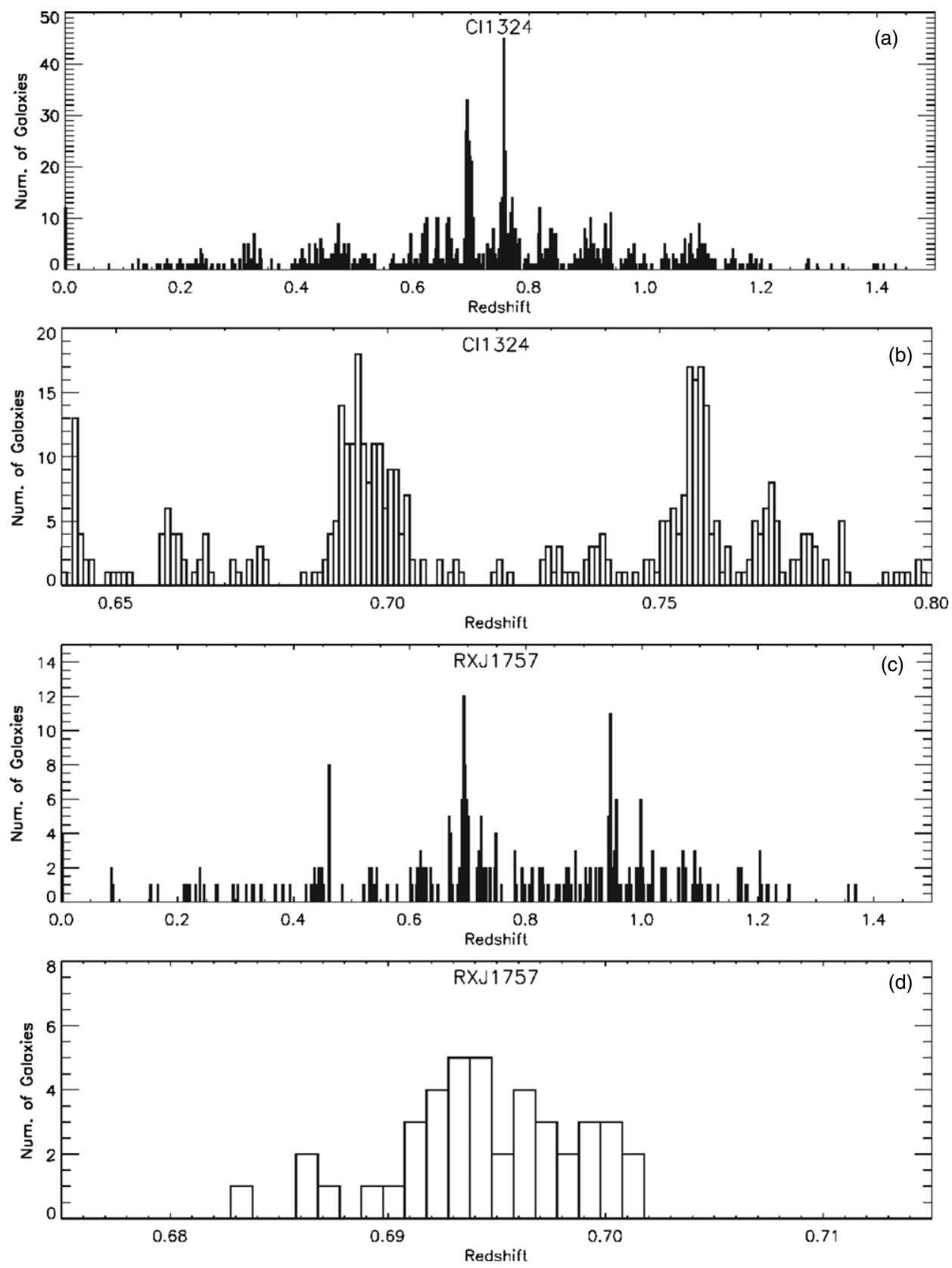

Figure 1. Redshift distributions for the C11324 supercluster and RXJ1757. The first two panels include (a) all measured redshifts for Cl1324, with $Q=3$ or 4 , up to $z=1.5$ and (b) zoomed in on the boundaries of the supercluster, $0.65<z<0.79$. The lower two panels include (c) all measured redshifts for RXJ1757, with $Q=3$ or 4 , up to $z=1.5$ and (d) zoomed in on the boundaries of the cluster, $0.68<z<0.71$. Although some $Q=3,4$ redshifts were measured above $z=1.5$, they have been omitted from these plots.

Table 5

C11324: Properties of Confirmed Groups and Clusters

\begin{tabular}{lcccc}
\hline \hline Name & $\begin{array}{c}\text { Alt. } \\
\text { Name }\end{array}$ & $\begin{array}{c}\text { R.A. } \\
(\text { J2000) }\end{array}$ & $\begin{array}{c}\text { Decl. } \\
(\text { J2000) }\end{array}$ & $\sigma^{\text {a }}$ \\
\hline Cl1324+3011 & A & 132448.7 & +301148 & $930 \pm 120$ \\
Cl1324+3059 & I & 132450.5 & +305819 & $870 \pm 120$ \\
Cl1324+3013 & B & 132421.5 & +301310 & $820 \pm 240$ \\
Cl1324+3025 & C & 132401.8 & +302505 & $220 \pm 100^{\text {b }}$ \\
\hline
\end{tabular}

Notes.

${ }^{\text {a }}$ Velocity dispersion measured in $\mathrm{km} \mathrm{s}^{-1}$, within $1 \mathrm{Mpc}$.

${ }^{\mathrm{b}}$ Dispersion calculated using only eight galaxies. distribution is reasonably consistent with a Gaussian, confirmed by a Kolmogorov-Smirnov (K-S) test at a 99\% level, suggesting there is no significant substructure. However, we caution that we have a smaller sample of confirmed redshifts compared to the other fields.

\subsection{The Red Sequence and the Blue Populations}

Figure 2 shows CMDs for all five fields. All spectroscopically confirmed supercluster/cluster members are shown. Squares indicate the confirmed X-ray AGNs within each structure, which are analyzed in Section 6. The red sequence for each field is delineated by dotted lines. Red-sequence fits for each field were calculated using a linear fitting and $\sigma$-clipping technique. First, 

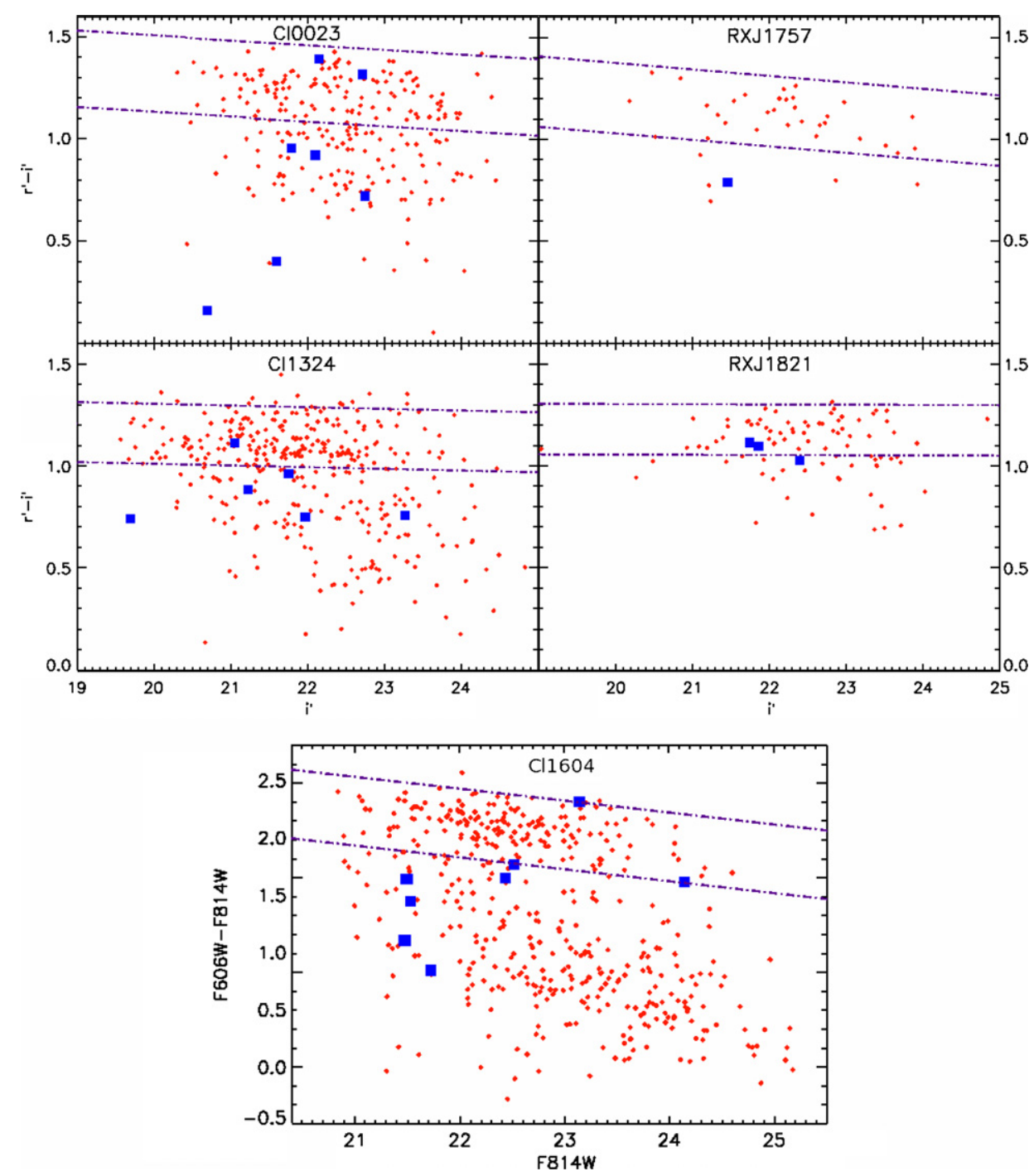

Figure 2. Color-magnitude diagrams for the five fields. LFC $r^{\prime}-i^{\prime}$ colors are displayed, except in the case of Cl1604, where ACS F606W-F814W colors are used. All confirmed supercluster/cluster members are shown and squares correspond to AGN hosts. Dashed lines indicate the boundaries of our red-sequence fits. We can see that a higher fraction of AGNs are on the red sequence in the more evolved structures (Cl1324, RXJ1821, and RXJ1757) than in Cl1604 and Cl0023.

(A color version of this figure is available in the online journal.)

a fit to a linear model, of the form

$$
C=C_{0}+m \times B
$$

where $C$ is either $r^{\prime}-i^{\prime}$ or F606W-F814W and $B$ is either $i^{\prime}$ or $\mathrm{F} 814 \mathrm{~W}$, was carried out on member galaxies within a chosen magnitude and color range using a $\chi^{2}$ minimization (Gladders et al. 1998; Stott et al. 2009). The fit was initialized with a color range chosen "by eye" to conform to the apparent width of the red sequence of the structure. The magnitude bounds were defined as the range where the photometric errors were small $\left(\sigma_{i^{\prime}, \mathrm{F} 814 \mathrm{~W}} \lesssim 0.05\right)$. After the initial fit, colors were normalized to remove the slope. The color distribution was then fit to a single Gaussian using iterative $3 \sigma$ clipping. At the conclusion of the algorithm, the boundaries of the red sequence were defined by a $3 \sigma$ offset from the center, except for $\mathrm{Cl} 1604$ and Cl1324. For the two superclusters, the color dispersion was inflated due to the large redshift extent of these structures, and $2 \sigma$ offsets were used to achieve reasonable boundaries. For every field except C11604, the LFC $r^{\prime}-i^{\prime}$ CMDs are shown. For C11604, ACS data were available and were used in place of LFC data because of their superior precision. Note, however, that two of the AGNs in
Cl1604 are outside our ACS pointings, so that our analysis using ACS data only includes eight AGNs in the Cl1604 supercluster.

While all the structures show a substantial number of galaxies on the red sequence, there are large differences in the blue populations. Qualitatively, we observe a lower blue fraction in the CMDs of RXJ1821 and RXJ1757 than in those of Cl0023 and Cl1604. This is quantified in Table 6, where the blue fraction ${ }^{10}$ for all confirmed cluster/supercluster members with $i^{\prime}$ or F814W magnitudes brighter than 23.5 are displayed in the first column. We can see that $\mathrm{Cl0023}$ and $\mathrm{Cl1604}$ have the bluest galaxy populations, while RXJ1757 has the highest fraction of galaxies on the red sequence of all the fields. Since this could be due to the low completeness of spectroscopic coverage in this field, we attempted to make corrections with two approaches: (1) using our efficiency of spectroscopically confirming structure members to estimate the total number of member galaxies and (2) correcting using measurements of the background galaxy density. All efforts to statistically estimate the true blue 10 We define a blue galaxy as any with a color blueward of the lower boundary
of the red sequence. 
Table 6

Global Blue Fractions

\begin{tabular}{lcc}
\hline \hline Structure & All Members & \\
\hline Cl0023 & $0.47 \pm 0.06$ & $0.51 \pm 0.13$ \\
Cl1604 & $0.57 \pm 0.05$ & $\ldots$ \\
Cl1324 & $0.42 \pm 0.04$ & $0.42 \pm 0.12$ \\
RXJ1821 & $0.35 \pm 0.08$ & $0.42 \pm 0.15$ \\
RXJ1757 & $0.17 \pm 0.08$ & $0.09 \pm 0.10$ \\
\hline
\end{tabular}

Notes.

a Includes all spectroscopically confirmed sources within the redshift bounds of the structure and with $i^{\prime}$ or F814W < 23.5. Errors are Poissonian.

${ }^{\mathrm{b}}$ Only spectroscopically confirmed sources from the first two spectral masks taken for each field are included, excluding masks where X-ray matched targets were preferentially targeted. For C11324, the first two masks from each pointing are included. No measurement was made for Cl1604. See Section 4.2 for explanation.

fraction yielded large errors making the measurements highly uncertain. Instead, we created a blue fraction measurement metric that could be compared between fields (see also Lubin et al. 2009). The different fields have a varying number of spectral masks, with several different spectroscopic priorities. However, the first several masks, excluding those designed to specifically target X-ray matched sources, have similar priorities for choosing targets. ${ }^{11}$ Therefore, we chose to compare blue fractions only among sources in the first two masks for each field, since these sources should represent similar populations. We chose two masks because this is the number of masks for RXJ1757 excluding those where objects matched to X-ray point sources were preferentially targeted. We confirmed that this sampling is representative of the entire galaxy population by recreating composite spectra using only the first two spectral masks. Since there are no large differences between the average spectral features, the method should be accurate. The results of this comparison are displayed in the second column of Table 6. However, we did not calculate a blue fraction for $\mathrm{Cl1604}$ in this way, for two reasons. First, there are a total of 24 spectroscopic masks for $\mathrm{Cl1604}$, from several different telescopes. Choosing which ones to include is difficult and it may be impossible to select a population congruous with any of the other fields in this manner. Second, our spectroscopy for Cl1604 is relatively complete, so we are confident in the blue fraction measured down to $\mathrm{F} 814 \mathrm{~W}=23.5$.

Examining the results, we can see the same color hierarchy in the structures for both methods of measuring the blue fraction, with RXJ1757 having the smallest fraction and Cl1604 and $\mathrm{Cl0023}$ the largest. These large blue fractions, in particular those for $\mathrm{Cl} 1604$ and $\mathrm{Cl} 0023$, are consistent with the Butcher-Oemler effect (Butcher \& Oemler 1984). We also note that the isolated X-ray-selected clusters, RXJ1757 and RXJ1821, are the reddest structures, suggestive of a more advanced, dynamically relaxed evolutionary state. The color hierarchy suggests a similar ranking of galactic star formation in the five structures, which we can explore with our spectroscopic data.

\subsection{Spectral Properties}

Using our spectroscopic data, we examine the typical star formation history of the galaxies in each structure. We formed

\footnotetext{
11 That is, prioritizing red galaxies first followed by progressively bluer
} galaxies (see, e.g., Gal et al. 2008; Lubin et al. 2009). composite spectra by co-adding the individual spectra of galaxies within each structure, according to the method of Lemaux et al. $(2009,2011)$. We analyze these spectra in terms of two important features relevant to star formation: the [O II] and $\mathrm{H} \delta$ lines. The $\mathrm{H} \delta$ absorption is indicative of a population of A and B stars, which disappears $\sim 1$ Gyr after the cessation of star formation within a galaxy, due to the lifetime of A stars (Poggianti \& Barbaro 1997). If star formation is ongoing, a population of $\mathrm{O}$ stars, which have weaker hydrogen features, can dominate the continuum and wash out this absorption line. Infilling can also occur from Balmer emission from $\mathrm{H}$ II regions. The [O II] emission line has been used as an indicator of star formation, especially as a proxy for the $\mathrm{H} \alpha$ emission line at higher redshifts when $\mathrm{H} \alpha$ has shifted out of the optical range (Poggianti et al. 1999). However, recent analysis using near-IR spectroscopy of sources from the $\mathrm{Cl1604}$ supercluster and RXJ1821 has compared [O II] and $\mathrm{H} \alpha$ emission, finding that a significant portion of [O II] emission can come from LINERor Seyfert-related processes (Lemaux et al. 2010; Kocevski et al. 2011a). These results are supported by those of Yan et al. (2006), albeit using a lower redshift sample. In light of this, caution must be exercised when interpreting [O II] measurements. For an additional diagnostic, we measure the $D_{\mathrm{n}}(4000)$ strength which is an indicator of mean stellar age (Kauffmann et al. 2003).

Figure 3(a) shows [O II] versus $\mathrm{H} \delta$ equivalent widths for the composite spectra of members of the five structures. The dotted line represents the average spectral properties for a cluster population composed of various fractions of "normal" starforming and quiescent galaxies (Dressler et al. 2004), based on data from the Two-Degree Field (2dF) Galaxy Redshift Survey (Colless et al. 2001). Asterisks on this line represent a cluster population composed of (from left to right) $20 \%, 40 \%$, $60 \%, 80 \%$, and $100 \%$ star-forming galaxies. For a cluster whose average galaxy lies above this line, the $\mathrm{H} \delta$ line is too strong to be produced by normal star formation, requiring some contribution from starbursting or post-starbursting galaxies. The dashed lines enclose $95 \%$ of the normal star-forming galaxies (Oemler et al. 2009). The shaded regions, which are based on the spectral types of Dressler et al. (1999) and Poggianti et al. (1999), denote the region of this phase space inhabited by (starting from the upper right and moving counterclockwise) starburst (dark blue), post-starburst (green), quiescent (red), and normal star-forming galaxies (light blue). Examining the positions of the structures in our sample on this plot, we can see that RXJ1757 and RXJ1821 have mostly quiescent populations, with each cluster having $\lesssim 20 \%$ normal star-forming galaxies. Because RXJ1757 is offset from the $2 \mathrm{dF}$ line, there may be some contribution from poststarburst galaxies, but the fractional contribution is low. The stronger [O II] emission in the $\mathrm{Cl0023}$ and $\mathrm{Cl1604}$ composite spectra suggests that these structures have a higher fraction ( $\gtrsim 40 \%$ ) of continuously star-forming galaxies. These structures have larger blue fractions than the others, so it is unlikely that the increased $\mathrm{EW}([\mathrm{O} \mathrm{II}])$ in their average spectra is due to LINER processes, which are primarily associated with redsequence galaxies. The $\mathrm{EW}(\mathrm{H} \delta)$ measured from the $\mathrm{Cl} 10023$ and Cl1604 composite spectra are significantly in excess of the $2 \mathrm{dF}$ line, suggesting a substantial contribution from starbursting or post-starburst galaxies. Cl1324 is in an intermediate range, with $\sim 30 \%$ normal star-forming galaxies and an observed $\mathrm{EW}(\mathrm{H} \delta)$ for its galaxy population smaller than that of $\mathrm{Cl} 10023$ and $\mathrm{Cl} 1604$.

Our conclusions based on the $[\mathrm{O} \mathrm{II}]$ and $\mathrm{H} \delta$ lines are supported by the corresponding $D_{\mathrm{n}}(4000)$ measurements (see Table 7). These results are illustrated in Figure 3(b), where 
Table 7

Composite Spectral Properties

\begin{tabular}{lccc}
\hline \hline Structure & $\begin{array}{c}\text { EW }([\mathrm{O} \text { II }])^{\mathrm{a}} \\
(\AA)\end{array}$ & $\begin{array}{c}\mathrm{EW}(\mathrm{H} \delta)^{\mathrm{a}} \\
(\AA)\end{array}$ & $D_{\mathrm{n}}(4000)^{\mathrm{b}}$ \\
\hline $\mathrm{Cl0023}$ & $-8.33 \pm 0.07$ & $3.17 \pm 0.05$ & $1.527 \pm 0.002$ \\
$\mathrm{Cl1604}$ & $-10.77 \pm 0.09$ & $2.99 \pm 0.07$ & $1.377 \pm 0.002$ \\
$\mathrm{Cl1324}$ & $-6.19 \pm 0.05$ & $2.40 \pm 0.03$ & $1.473 \pm 0.001$ \\
RXJ1821 & $-4.20 \pm 0.10$ & $1.39 \pm 0.08$ & $1.631 \pm 0.004$ \\
RXJ1757 & $-1.87 \pm 0.18$ & $1.77 \pm 0.10$ & $1.591 \pm 0.005$ \\
\hline
\end{tabular}

Notes.

${ }^{a}$ Measured using bandpasses from Fisher et al. (1998).

b Measured using bandpasses from Balogh et al. (1999).

average measurements of $\mathrm{EW}(\mathrm{H} \delta)$ and $D_{\mathrm{n}}(4000)$ are plotted for the five structures. For comparison, we also indicate ranges of $\mathrm{EW}(\mathrm{H} \delta)-D_{\mathrm{n}}(4000)$ phase space spanned by four different Bruzual \& Charlot (Bruzual 2007) models for various times after the starburst. The four models include a single burst $(\tau=0.01)$ and a secondary burst of $20 \%, 10 \%$, and $5 \%$ by mass which occurs 2 Gyr after the initial burst. All models have solar metallicity and are corrected for extinction using $E(B-V)=0.25$ and a Calzetti et al. (2000) extinction law. Because Bruzual \& Charlot models only incorporate stellar light, emission infill corrections were made for all $\mathrm{EW}(\mathrm{H} \delta)$ measurements using relationships between $\mathrm{H} \alpha$ and [O II] taken from Yan et al. (2006) and $\mathrm{H} \alpha$ and $\mathrm{H} \delta$ from Schiavon et al. (2006). Although these corrections are not perfect (e.g., see the measurement for Region 1 ), these values should be accurate to within $\pm 0.5 \AA$. We can see that RXJ1757 and RXJ1821 have the largest continuum break strengths, indicating that they possess the oldest average stellar populations of the five structures. The average galaxy in these structures has had 1-3 Gyr since its last starburst, according to the Bruzual (2007) model. The other structures have smaller average $D_{\mathrm{n}}(4000)$ measurements, consistent with the results of Figure 3(a) showing larger fractions of star-forming galaxies and younger galaxy populations. According to the Bruzual (2007) model, the average galaxy in $\mathrm{Cl1324,} \mathrm{Cl0023}$, and $\mathrm{Cl1604}$ has had a progressively shorter time since the last starburst. Altogether, these spectral results parallel what was found with the blue fractions, in that the reddest structures are also the ones with the most evolved stellar populations and the lowest fraction of star-forming galaxies.

\subsection{Summary of Global Characteristics and Grouping of the Sample}

Despite our extensive spectroscopic sample, there are too few $\mathrm{X}$-ray AGNs in any individual cluster (and even supercluster) to draw statistical conclusions. Hence, we divide our structure sample into two categories. The first contains C10023 and Cl1604 which have the highest level of ongoing star formation and starburst activity, as shown in the preceding sections. The second category, consisting of Cl1324, RXJ1757, and RXJ1821, contains structures whose member galaxies are typically quiescent or forming stars at a lower rate. We refer to these two categories as "unevolved" and "evolved," respectively, as a means of describing their typical galaxy populations. While we acknowledge that the terms "more evolved" and "less evolved" would be more appropriate, we adopt the less accurate denominations for brevity. In addition, these terms do not necessarily imply differing levels of cluster dynamical evolution or even a clear temporal sequence from one category to the other. We note that, while some clusters or groups may not fit well with the global characteristics of their parent supercluster (i.e., Cluster A of C11604), we cannot examine all of the AGNs on a clusterby-cluster basis. As we will discuss in Section 6.1, many of the AGNs in the superclusters and the supergroup are not associated with any one cluster or group. So, when analyzing these AGNs, we take the parent supercluster or supergroup as a whole.

The particular segregation of our structure sample is motivated by the clear distinction between the structures shown in Figure 3. The abundant star formation in the unevolved structures suggests the presence of a large gas reservoir in many of their member galaxies. We might expect that this same gas is available for AGN fueling. Conversely, the typical galaxy in the evolved sample has likely consumed most of the available gas in prior star formation episodes, leaving less fuel for the AGNs. In the following sections, we examine whether the properties of the AGNs in the two categories reflect this distinction, and what we can learn about the relationship of star formation to AGN activity in LSSs.

\section{CUMULATIVE X-RAY SOURCE COUNTS}

In this section, we examine the frequency of AGN activity within the five structures to determine if there are associated excesses of X-ray point sources. X-ray point source photometry was used to calculate cumulative X-ray source number counts, $N(>S)$, using the method of Gioia et al. (1990):

$$
N(>S)=\sum_{i=0}^{N} \Omega_{i}^{-1} \operatorname{deg}^{-2},
$$

where $N$ is the number of point sources with fluxes greater than $S$ and $\Omega_{i}$ is the area of the sky in which the $i$ th point source could have been detected at a $3 \sigma$ level or higher. Variance in $N(>S)$ was calculated using $\sigma_{i}^{2}=\sum_{i=0}^{N} \Omega_{i}^{-2}$.

To calculate $\Omega_{i}$, we used the method of Kocevski et al. (2009a), which is similar to those used in other literature (Johnson et al. 2003; Cappelluti et al. 2005). All point sources detected by wavdetect, in all bands and without significance cuts, were removed and replaced with an estimate of the background using the CIAO tool dmfilth. To create a map of the background emission, these images were binned into $32^{\prime \prime}$ bins. According to Equation (1), the flux limit corresponding to a $3 \sigma$ detection $^{12}$ occurring in one of these binned pixels is given by

$$
S_{\mathrm{lim}}=3 \frac{k}{t}\left(1.0+\sqrt{0.75+B \pi R_{95}^{2} A^{-1}}\right),
$$

where $B$ is the net counts in a pixel, $A$ is the area of a pixel, $R_{95}$ is the radius of the aperture enclosing $95 \%$ of an X-ray source's flux, as described in Section $3.3, k$ is the conversion factor between photon count rate and X-ray flux, also described in Section 3.3, and $t$ is the exposure time of the image. For a given source with flux $S, \Omega_{i}$ is then equal to the total number of binned pixels for which $S$ is greater than $S_{\text {lim }}$, multiplied by the area of a pixel.

The cumulative source counts for all the fields in the soft band and the 2-10 keV band, hereafter the hard ${ }_{10}$ band, are shown in Figure 4. The latter was extrapolated, field by field, from

\footnotetext{
12 Note that we only include X-ray sources with detection significances $>3 \sigma$ in this analysis to be consistent with previous work. The inclusion of sources with detection significances between $2 \sigma$ and $3 \sigma$ has a significant effect only on fluxes where sky coverage is below $20 \%$, and the results are generally considered unreliable.
} 

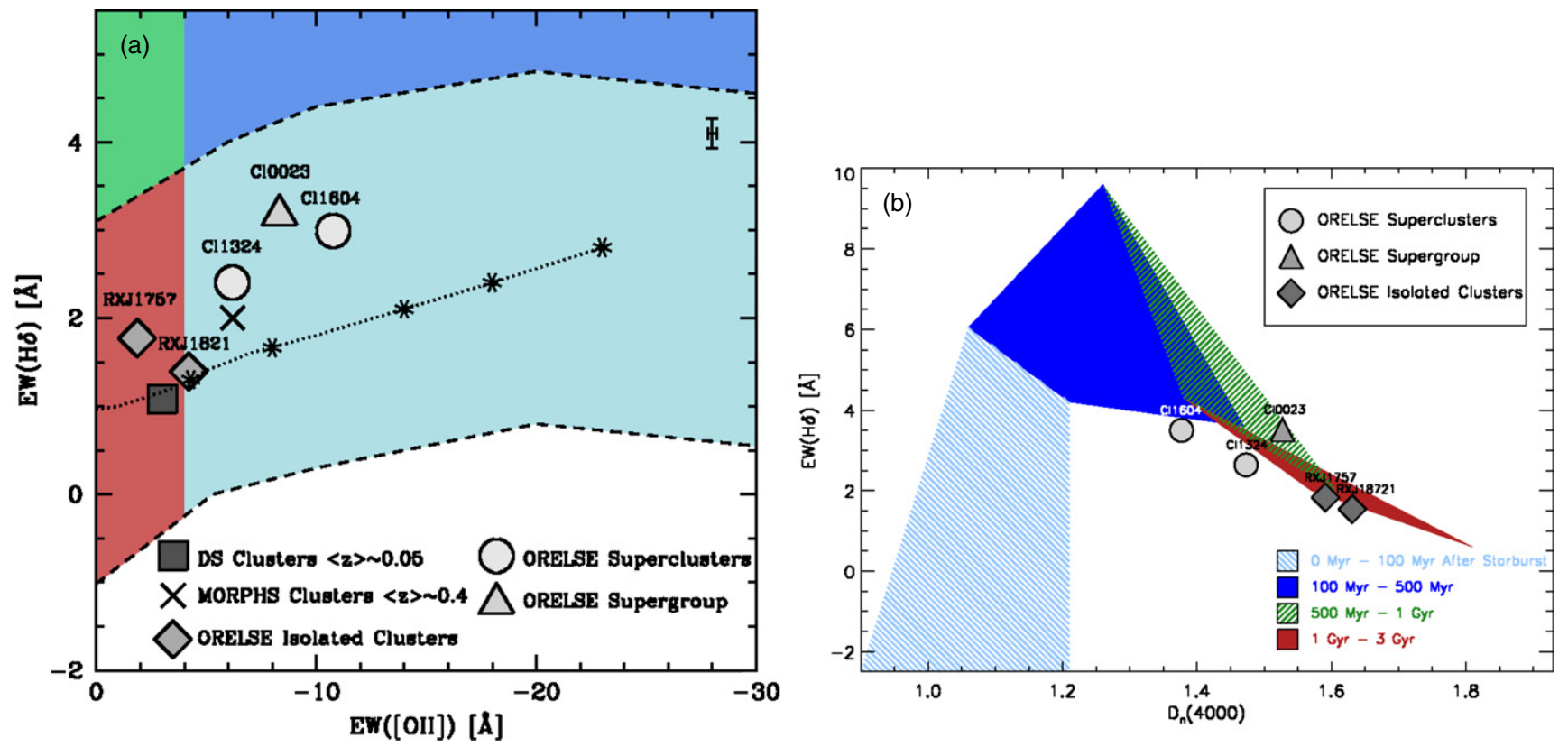

Figure 3. Spectral properties of the composite spectra of all spectroscopically confirmed galaxies in our five structures. In (a), EW([O II]) is used as a measure of star formation while $\mathrm{EW}(\mathrm{H} \delta$ ) is used to probe the post-starburst stellar population (Poggianti \& Barbaro 1997; Poggianti et al. 1999). The dotted line is a track for clusters made entirely of quiescent and continuously star-forming galaxies from $2 \mathrm{dF}$ (Dressler et al. 2004), with a lower percentage of star-forming galaxies to the left. The shaded regions represent galactic classifications as described in the text. The dashed lines enclose 95\% of the normal star-forming galaxies (Oemler et al. 2009). Spectral errors are shown in the upper right-hand corner. Also shown, for reference, are samples of cluster galaxies at $z \sim 0.05$ (Dressler \& Shectman 1988) and $z \sim 0.4$ (Dressler et al. 2004). In (b), we plot the average $D_{\mathrm{n}}(4000)$ measurements of structure members vs. the average $\mathrm{EW}(\mathrm{H} \delta)$. For reference, we plot regions of $\mathrm{EW}(\mathrm{H} \delta)-D_{\mathrm{n}}(4000)$ phase space covered by four post-starburst models of Bruzual (2007). Four different time ranges since starburst are shown. Because Bruzual \& Charlot models only incorporate stellar light, emission infill corrections were made for all $\mathrm{EW}(\mathrm{H} \delta)$ measurements (see Section 4.3 for details). $D_{\mathrm{n}}(4000)$ and $\mathrm{EW}(\mathrm{H} \delta)$ errors are smaller than the points.

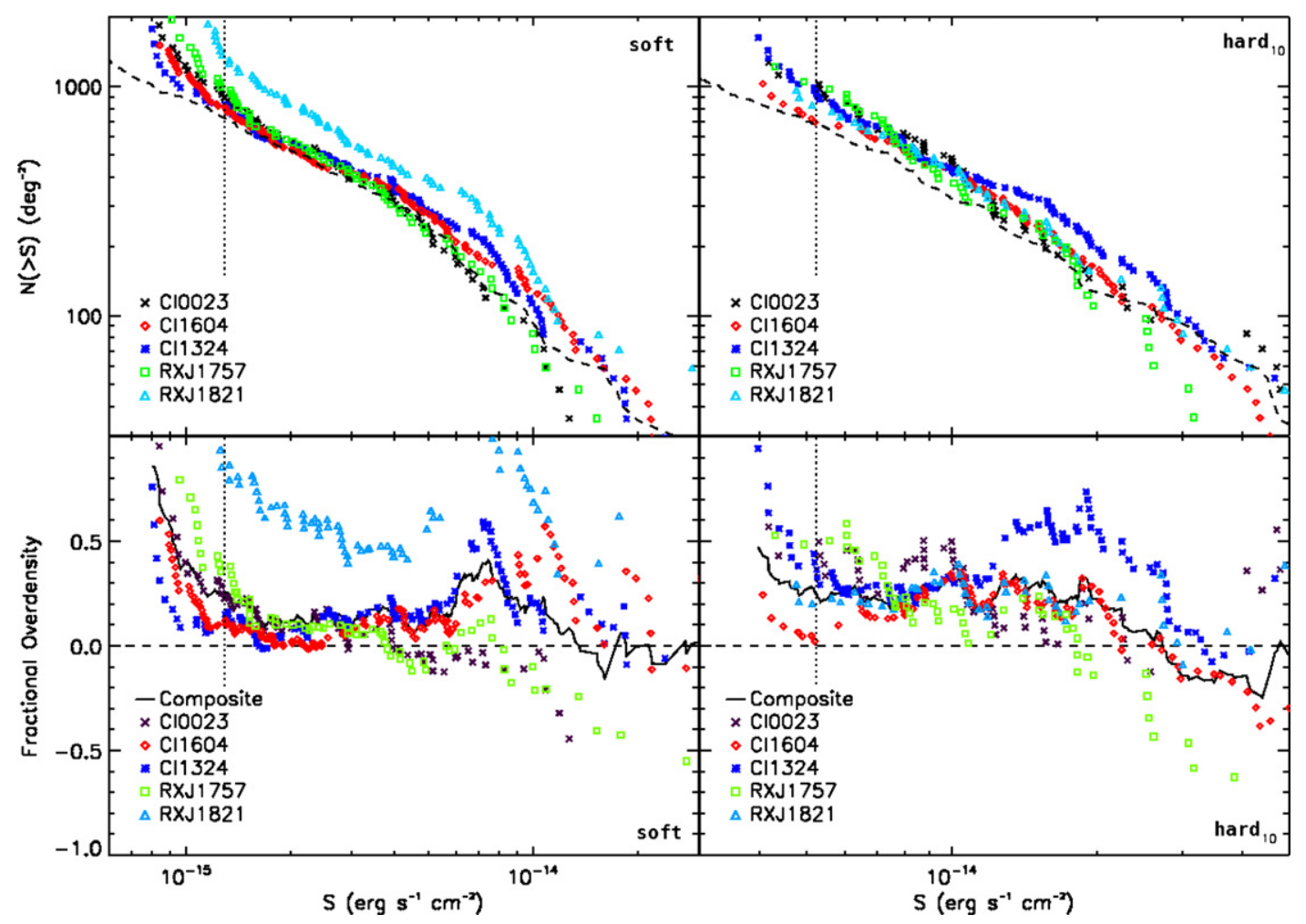

Figure 4. Cumulative source counts for all five structures, in the soft $(0.5-2.0 \mathrm{keV})$ band (top left) and hard 10 (2.0-10 keV) band (top right). The dashed line corresponds to source counts from two pointings in the CDFN and CDFS, which we reduced and analyzed ourselves. The dotted line indicates flux corresponding to $20 \%$ sky coverage for NEP5281 in the soft band and RXJ1757 in the hard band. Note that this value is different for each field and the specific choice of field here is meant to be representative. In the lower plots, we have plotted the fractional overdensities relative to the CDF deep field measurements. Along with the five fields is a composite measurement, explained in Section 5. 


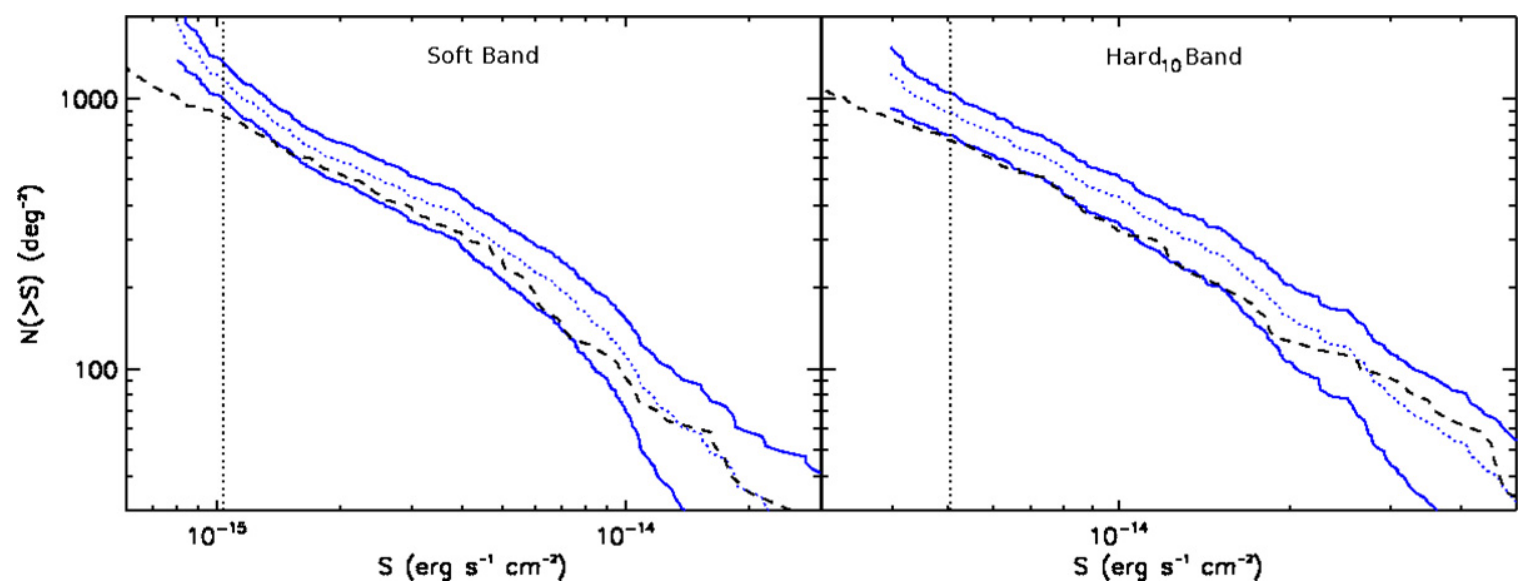

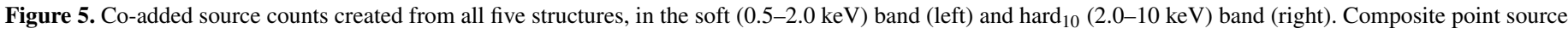

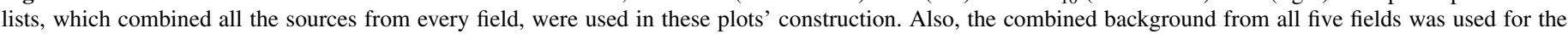

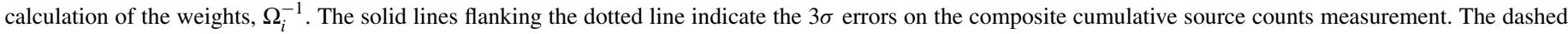

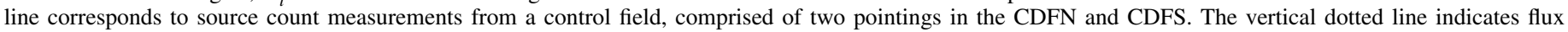
corresponding to $20 \%$ sky coverage of the combined fields.

(A color version of this figure is available in the online journal.)

the $2-8 \mathrm{keV}$ band by fitting a power-law spectral model with exponent $\gamma=1.4$ to each detected point source. For $\mathrm{Cl} 1604$ and Cl1324, the source counts for the two pointings were combined. Also shown are cumulative source count measurements from the Chandra Deep Field North and South (CDFN and CDFS; Brandt et al. 2001; Rosati et al. 2002). One Chandra observation from each deep field was used (observation IDs 582 and 2232). Both have exposure times of roughly $130 \mathrm{ks}$. We re-analyzed these observations using the same reduction pipeline that we used for the ORELSE fields. We used the combined source counts from these two fields (hereafter CDF) to estimate the blank-field counts for comparison with our data.

We can see that, in the soft band, C10023 and RXJ1757 appear to be consistent with no overdensity compared to CDF. The other three fields are all overdense to some degree. In the range $3 \times 10^{-15}$ to $10^{-14} \mathrm{erg} \mathrm{s}^{-1} \mathrm{~cm}^{-2}, \mathrm{RXJ} 1821, \mathrm{Cl1324}$, and Cl1604 have average overdensities of $0.5 \sigma, 1 \sigma$, and $1.5 \sigma$, respectively, with $\sigma$ calculated using the cumulative source count errors from our data and those of CDF. In the hard 10 band, RXJ1821, RXJ1757, and $\mathrm{Cl} 0023$ all have approximate overdensities of $0.5 \sigma$ in the range $7 \times 10^{-15}$ to $2 \times 10^{-14} \mathrm{erg} \mathrm{s}^{-1} \mathrm{~cm}^{-2}$. RXJ1757 appears to be consistent with no overdensity. Cl1604 and Cl1324 also appear to be overdense, with average overdensities in the flux range $7 \times 10^{-15}$ to $2 \times 10^{-14} \mathrm{erg} \mathrm{s}^{-1} \mathrm{~cm}^{-2}$ of $1 \sigma$ and $1.5 \sigma$, respectively.

It should be noted that the results for $\mathrm{Cl} 0023$ differ from those presented in the previous work of Kocevski et al. (2009c). The 2-8 keV band cumulative source counts were unintentionally presented as the $2-10 \mathrm{keV}$ band counts in that paper. However, we have also found changes due to the different versions of the CIAO software used among earlier papers (Kocevski et al. 2009a, 2009b, 2009c) and this paper, version 3.3 compared to 4.2. We believe this to be an effect of updated response functions at off-axis angles. The net result for our data is mainly an approximate $5 \%$ increase in flux, with some very minor differences in point source detection, the latter being almost entirely below the $3 \sigma$ level.

In Figure 5, co-added cumulative source counts for all five fields are shown. To accomplish this co-addition, all point source lists were first combined into one composite source list. For the calculation of the effective sky area weighting factors, $\Omega_{i}$, the combined background of every field was used. With $\sigma_{i}^{2}=\sum_{i=0}^{N} 1 / \Omega_{i}^{2}$, we can see that the error in $N(>S)$ will be reduced from its value in any individual field. In the soft band, the composite source counts have an approximate average overdensity of $1 \sigma$ in the range $2 \times$ $10^{-15}$ to $8 \times 10^{-15} \mathrm{erg} \mathrm{s}^{-1} \mathrm{~cm}^{-2}$, which falls off to zero brighter than $10^{-14} \mathrm{erg} \mathrm{s} \mathrm{sm}^{-1}$. In the hard $\mathrm{cm}_{10}$ band, the composite counts have an approximate average overdensity of $1 \sigma$ between $7 \times 10^{-15}$ and $2 \times 10^{-14} \mathrm{erg} \mathrm{s}^{-1} \mathrm{~cm}^{-2}$, but an approximate underdensity of $0.5 \sigma$ between $3 \times 10^{-14}$ and $4 \times 10^{-14} \mathrm{erg} \mathrm{s}^{-1} \mathrm{~cm}^{-2}$. While there is substantial variation in the five cumulative source count measurements for the individual fields, we can see from the composite measurement that, on average, the fields of the five structures studied here have a density of X-ray point sources in excess of the control field, though not significantly so.

Cappelluti et al. (2005, hereafter C05) have found a positive correlation between cluster redshift and source count overdensity. To compare to this, and our earlier results of Kocevski et al. (2009a), we first fit the cumulative source counts to a power law of the form $N(>S)=k\left(S / S_{0}\right)^{-\alpha} \mathrm{deg}^{-2}$, using the maximum likelihood method of Crawford et al. (1970) and Murdoch et al. (1973). This method fixes the dimensionless variable $k$ while fitting for $\alpha$. $k$ is calculated by requiring consistency between the model and the data at the flux point $S=S_{0}$. We use $2 \times 10^{-15} \mathrm{erg} \mathrm{s}^{-1} \mathrm{~cm}^{-2}$ and $1 \times 10^{-14} \mathrm{erg} \mathrm{s}^{-1} \mathrm{~cm}^{-2}$ for $S_{0}$ in the soft and hard ${ }_{10}$ bands, respectively, which are the values used by Crawford et al. (1970). C05 measured overdensities using the ratio of $k$ in a given field to $k$ in a reference set of five blank fields. For these fields, they found $k_{\text {soft }}=401_{-13}^{+31}$ and $k_{\text {hard }}=275_{-9}^{+22}$. We calculated $k$ for the five fields individually, as well as the composite of all five fields. It should be noted that the composite measurement is not a simple average of the five fields (see above for details on its creation) and that significant variation in the individual $k$ values come from calculating $k$ at a single flux point.

Our results are shown in Figure 6 for the hard ${ }_{10}$ band, where our data are overplotted on that of $\mathrm{C} 05$ and the linear fit to their data is shown. Just as in Kocevski et al. (2009a), our 


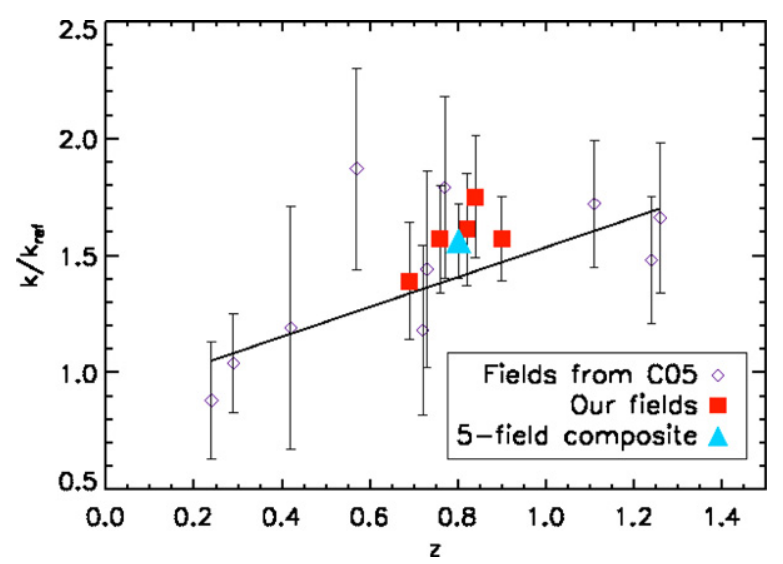

Figure 6. Overdensity amplitude vs. redshift in the hard 10 band, as defined by Cappelluti et al. (2005). $k$ is defined as the value of $N(>S)$ at $S_{0}=$ $10^{-14} \mathrm{erg} \mathrm{s}^{-1} \mathrm{~cm}^{-2}$. Shown are the ten fields analyzed by $\mathrm{C} 05$ and their fit to this data set. Our fields are overplotted as squares, along with a composite measurement made by co-adding all five fields. (Adapted from Figure 6 of C05.) (A color version of this figure is available in the online journal.)

data are consistent with their fit. Once again (refer to earlier explanation), it is possible that the issue with the version of the CIAO software may have created a systematic offset from $\mathrm{C} 05$ 's data set. We would expect this offset to increase the value of $k$ for the $\mathrm{CO} 5$ data by about $5 \%$. Even without this correction, our results are still consistent with $\mathrm{C} 05$ within our errors. However, we note that it is difficult to use these overdensities to interpret the actual AGN activity in an individual structure, even with our large spectroscopic sample (see Section 6).

\section{AGN AND HOST PROPERTIES}

Using optical sources with redshifts with quality flags of $Q=3$ or 4 and the results of our optical matching, we were able to identify X-ray sources that are members of the clusters or superclusters in our sample (see Table 3). In summary, we found ten confirmed AGNs in the bounds of Cl1604, seven in Cl0023, six in C11324, three in RXJ1821, and one in RXJ1757. Note that these numbers include four sources, one in each structure except RXJ1757, that were detected at a $<3 \sigma$ (but at a $>2 \sigma$ ) level in at least one of the three X-ray passbands (see Table 8). We show in the following that these low-significance detections do not bias our results.

\subsection{Spatial Distribution}

Examining the spatial distribution of AGNs located within each cluster can give insight into what processes triggered their nuclear activity. In Figures 7, 8, and 9, we show the spatial distributions on the sky and redshift distributions of the five structures studied here. The AGNs are marked in red, and their positions and characteristics are given in Table 8 .

Of particular note is the lack of AGNs in dense cluster centers. Indeed, we find $19 \%$ of AGNs in cluster cores, defined as being within a projected distance of $0.5 \mathrm{Mpc}$ to the nearest cluster or group. ${ }^{13}$ An additional $27 \%$ lie on the outskirts of clusters (projected distances between 0.5 and $1.5 \mathrm{Mpc}$ ), and a majority ( $>50 \%$ ) of AGN host galaxies lie more than $1.5 \mathrm{Mpc}$ in projected distance from the nearest cluster or group.

\footnotetext{
${ }^{13}$ Redshift is taken into account when determining proximity of AGNs to clusters or groups in the superclusters and the supergroup. Projected two-dimensional distance is only calculated to clusters of similar redshift.
}

These results are consistent with previous work studying the spatial distribution of AGNs, in that the AGNs tend to be located outside of clusters or in their outskirts. First, the distribution of AGNs in Cl1604, previously studied by Kocevski et al. (2009a), is consistent with the other four structures here. Our results are also consistent with those of Gilmour et al. (2007), who found that AGNs in the A901/902 supercluster at $z \approx 0.17$ tend to avoid the densest areas. Several other studies have also found that X-ray AGNs tend to reside in regions of moderate density similar to group environments, up to $z \sim 1$ (Hickox et al. 2009; Silverman et al. 2009a). It is thought that regions of intermediate density, such as the outskirts of clusters, are the most conducive to galaxy-galaxy interactions because of their elevated densities, compared to the field, but relatively low velocities (Cavaliere et al. 1992). Since we find more of the AGNs in these areas, this lends support to the theory that mergers or tidal interactions are one of the main instigators of AGN activity. Since only 20\% inhabit dense cluster cores, processes which preferentially occur in these regions, such as ram pressure stripping, are probably not responsible for triggering AGNs in cluster galaxies. However, the association between AGNs and these regions could also be related to higher gas availability in galaxies farther from cluster cores.

Although we find that most of the X-ray AGNs do not reside in the cluster cores, a number of studies have measured the fraction of cluster galaxies that host AGNs (e.g., Martini et al. 2007). Therefore, we attempt to measure the AGN fraction for the individual clusters within the five structures in our sample. Because we are limited by the spectral completeness of our sample, we make a composite measurement of the most massive, well-sampled clusters: C11604A, C11604B, Cl1604C, Cl1604D, Cl1324A, Cl1324I, and RXJ1821. We compare to the results of Martini et al. (2007) for low-redshift clusters $(0.06<z<0.31)$, who used galaxies with $M_{R}<-20$, within approximately $2000 \mathrm{~km} \mathrm{~s}^{-1}$ of the mean redshift of cluster members for each system, and within the field of view of their Chandra observations, which ranged from 1.2 to $4.5 \mathrm{Mpc}$ in width. To approximate these criteria, we adopt a magnitude cutoff that roughly corresponds to $M_{i^{\prime} / \mathrm{F} 814 \mathrm{~W}}=-19$ and use galaxies within $1 \mathrm{Mpc}$ and $\Delta z=0.01$ of the cluster centers and redshifts. We measure the combined AGN fraction for the seven clusters listed above to be $0.012 \pm 0.007$. This is consistent with the results of Martini et al. (2007), who measured $f_{A}\left(M_{R}<-20 ; L_{x}>10^{42} h_{70}^{-2} \mathrm{erg} \mathrm{s}^{-1}\right) \approx 1 \%$. We note, however, that a number of arbitrary definitions went into our measurement. In addition, our spectroscopy of optical counterparts to X-ray sources is significantly incomplete, in contrast to Martini et al. (2007). Correcting for this incompleteness contributes the largest source of error to our measurement. Because of the large uncertainties, we refrain from drawing any conclusions from our measurement.

\subsection{AGN Host Galaxy Colors}

Figure 2 presents CMDs, which are described in Section 4, for all five fields. Confirmed AGN members of the structures are shown with blue squares. While the AGNs in C11324, RXJ1821, and RXJ1757 preferentially reside within the bounds of, or very close to, the red sequence, the AGN hosts in $\mathrm{Cl} 10023$ and $\mathrm{Cl} 1604$ are more spread out. Previous work, including Kocevski et al. (2009a) and a number of wide-field surveys (Sánchez et al. 2004; Nandra et al. 2007; Georgakakis et al. 2008; Silverman et al. 2008), has found an association between AGN activity in galaxies and the transition onto the red sequence (possibly 

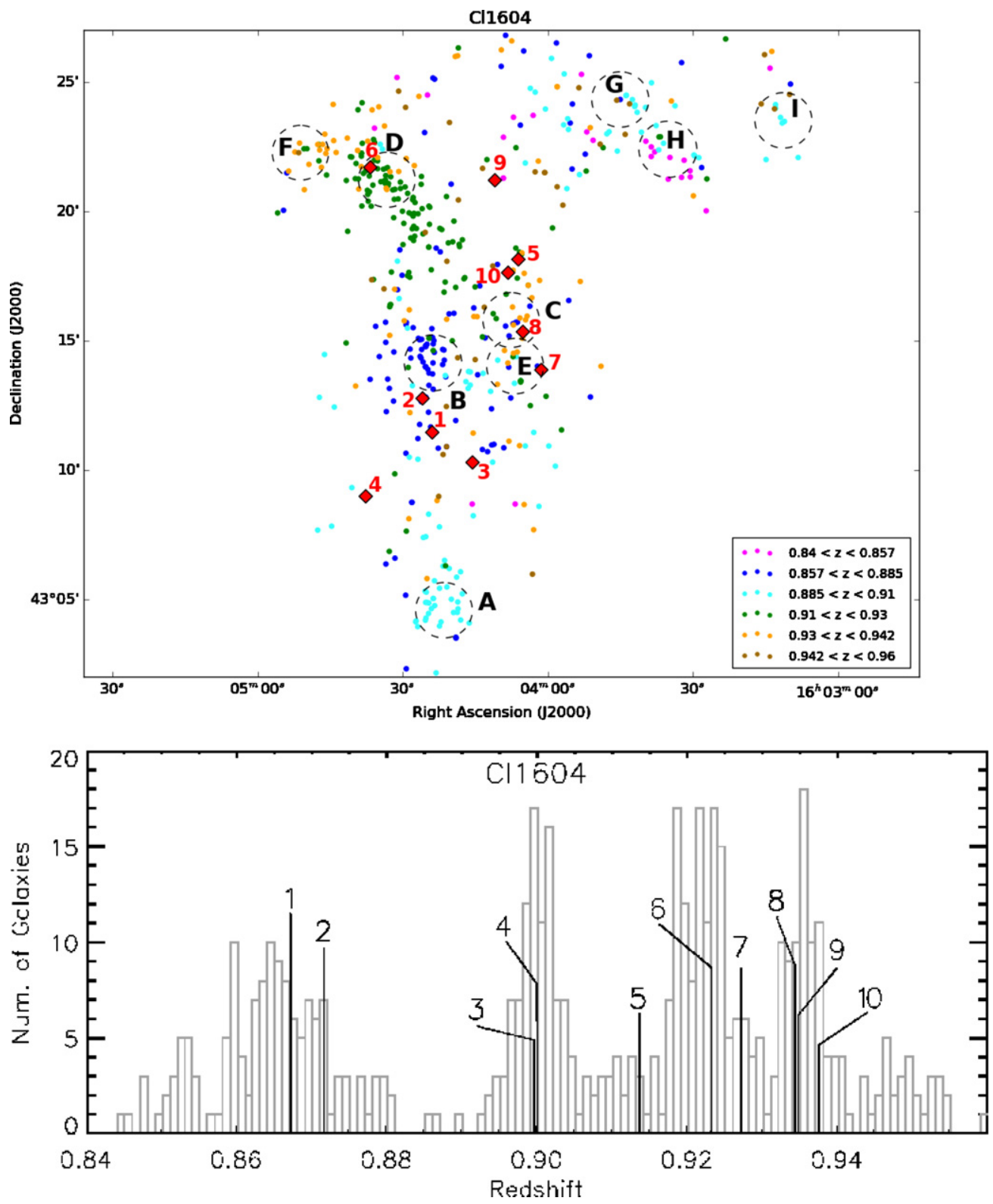

Figure 7. Spatial map and redshift distribution for Cl1604, providing a three-dimensional map of the structure. All optical sources within the redshift bounds of the supercluster are shown, and are color-coded according to redshift. Confirmed AGNs are plotted on the spatial maps in red, and are also shown in the redshift distributions. Dashed circles show all clusters and groups in the structure, although E is not confirmed, and have radii of $0.5 h_{70}^{-1} \mathrm{Mpc}$ at their respective redshifts.

for a second time) in the green valley. While these galaxies could be evolving from the blue cloud onto the red sequence, it is also possible that they could have moved down off the red sequence after a tidal interaction or merger and are evolving back (Menanteau et al. 2001; Kocevski et al. 2009a). We note, however, that some studies using mass-selected samples have that AGN hosts have found a color distribution more similar to that of normal galaxies (Silverman et al. 2009b; Xue et al. 2010).

In addition, Cardamone et al. (2010), using a sample of galaxies with redshifts $0.8<z<1.2$, have found that many green-valley AGN hosts are dust-reddened blue cloud members, so that AGN host colors acquire the bimodality apparent in the general galaxy population. However, Rosario et al. (2011) have also examined the impact of extinction on the colors of AGN hosts and did not find a significant impact for galaxies in the redshift range $0.8<z<1.2$, although bimodality may be introduced at higher redshifts. To address this issue for our study, we are planning to implement spectral energy distribution (SED) fitting to evaluate the impact of extinction on the broadband colors of our sample. Preliminary results from SED fitting of the $\mathrm{Cl1604}$ hosts suggest that extinction levels in our sample are not as drastic as those presented by Cardamone et al. (2010). It is also possible that AGN host colors are contaminated by the AGNs themselves. However, this is unlikely because (1) almost all AGN hosts in our sample have rest-frame X-ray luminosities below the quasistellar object (QSO) level of $10^{44} \mathrm{erg} \mathrm{s}^{-1}$ (see Section 6.4) and (2) Kocevski et al. (2009b) found that, in C11604, AGN hosts with blue cores did not have a rising blue continuum indicative of QSO activity. Therefore, we proceed to investigate the AGN association with the transition zone and to explore differences in the evolutionary states of their host galaxies in each field by examining color offsets of the AGN hosts from the red sequence.

Histograms of offsets from the center of the red sequence are shown in Figure 10. The first two panels present offsets in terms of color. In the top panel, only the structures where LFC 

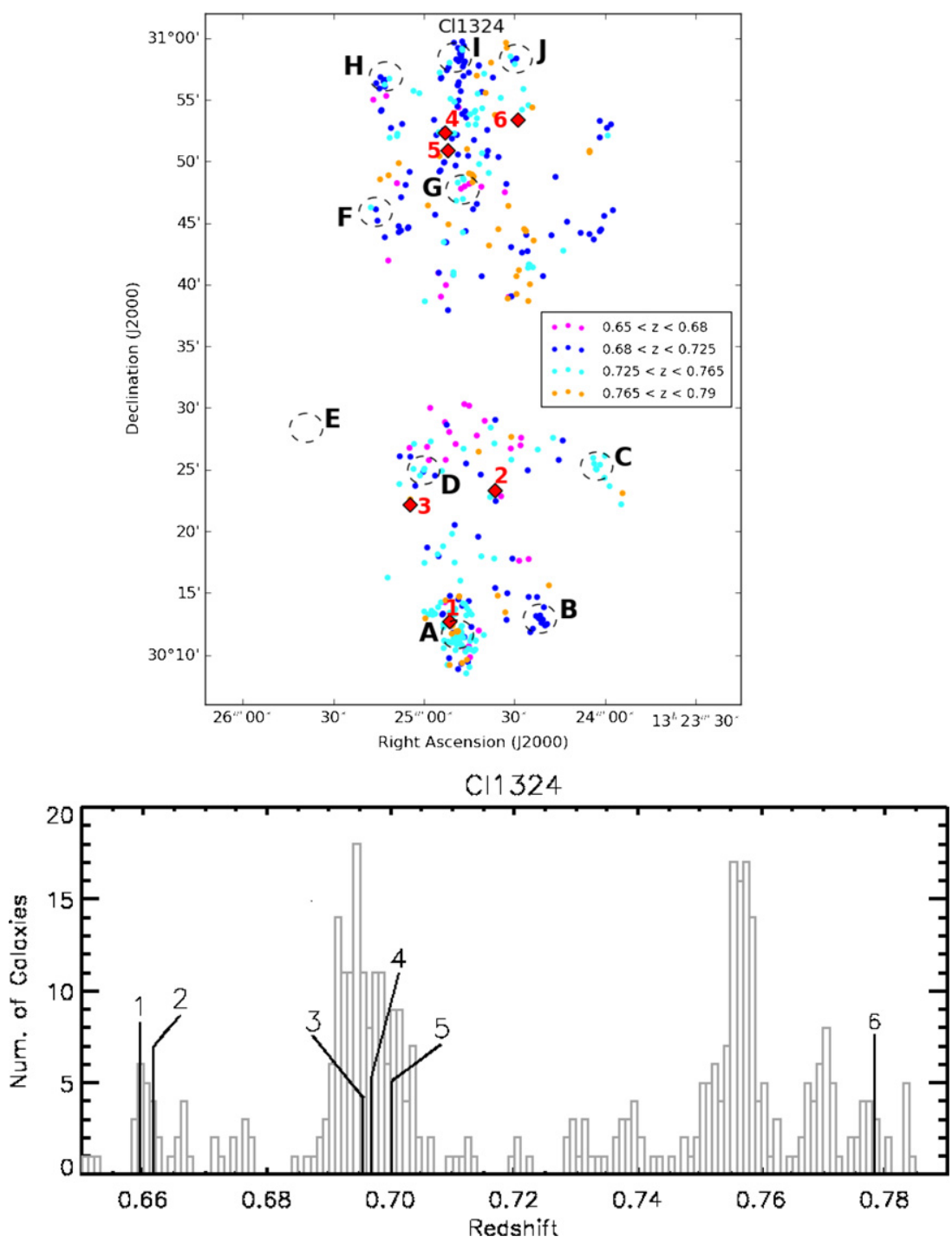

Figure 8. Spatial map and redshift distribution for Cl1324. All optical sources within the redshift bounds of the supercluster are shown, and are color-coded according to redshift. Confirmed AGNs are plotted on the spatial maps in red, and are also shown in the redshift distributions. Dashed circles show all clusters and groups in the structure and have radii of $0.5 h_{70}^{-1} \mathrm{Mpc}$ at their respective redshifts. These are derived from red galaxy overdensities, and only Clusters A, B, D, and I have been confirmed (see R. R. Gal et al. 2012, in preparation).

data were used are shown, which is every field except Cl1604. The middle panel shows only $\mathrm{Cl1604}$, for which we used ACS colors. In the bottom panel, all five structures are shown, with normalized offsets. In order to compare the ACS and LFC data, we scale by the red-sequence width, $W_{\mathrm{RS}}$. We define $W_{\mathrm{RS}}$ as the distance from the center of the red-sequence fit to its boundary (see Section 4.2 and Figure 2). On this plot, AGN hosts on the red sequence will then be located between -1.0 and 1.0.

With red-sequence offsets, we can quantitatively examine the green valley. In Figure 10(b), we plot a histogram of RS offsets, measured from the ACS data, for the AGNs in the Cl1604 supercluster. For comparison, we overplot a scaled distribution of RS offsets for all spectroscopically confirmed supercluster members with ACS photometry. In the scaled histogram, we can clearly see an area of reduced number density between the red sequence and blue cloud. For C11604, the green valley can be approximated as the region $-3 W_{\mathrm{RS}}<\Delta C<-W_{\mathrm{RS}}$, where $\Delta C$ is the offset of an AGN host from the center of the red sequence. Only $\sim 17 \%$ of all confirmed supercluster members with ACS data fall within this region. However, five out of eight of the C11604 AGN hosts with ACS data reside within it. While it is unclear how well this definition of the green valley extends to LFC data, because of larger photometric errors, we can see in Figure $10(\mathrm{c})$ that $36 \%$ of all host galaxies have $-2 W_{\mathrm{RS}}<\Delta C<-W_{\mathrm{RS}}$ and $60 \%$ are in the range $-3 W_{\mathrm{RS}}<\Delta C<-W_{\mathrm{RS}}$.

While many galaxies in both the evolved and unevolved structures lie in the "green valley" region, the percentage of AGNs on the red sequence is somewhat higher in the evolved structures compared to the unevolved structures, with $30 \%$ and $20 \%$, respectively. Examining Figure 10, we can see the distribution of AGN hosts in evolved structures is clustered closer to the red sequence, while in the unevolved structures, this distribution has a large tail extending into the blue cloud. Indeed, none of the AGN host galaxies in the evolved structures have $\Delta C<-3 W_{\mathrm{RS}}$, whereas four of the X-ray AGN hosts in the unevolved structures have red-sequence offsets below this limit. Although these results are suggestive (and unaffected by our inclusion of the $<3 \sigma \mathrm{X}$-ray sources), the two distributions are not statistically different based on the K-S test. 

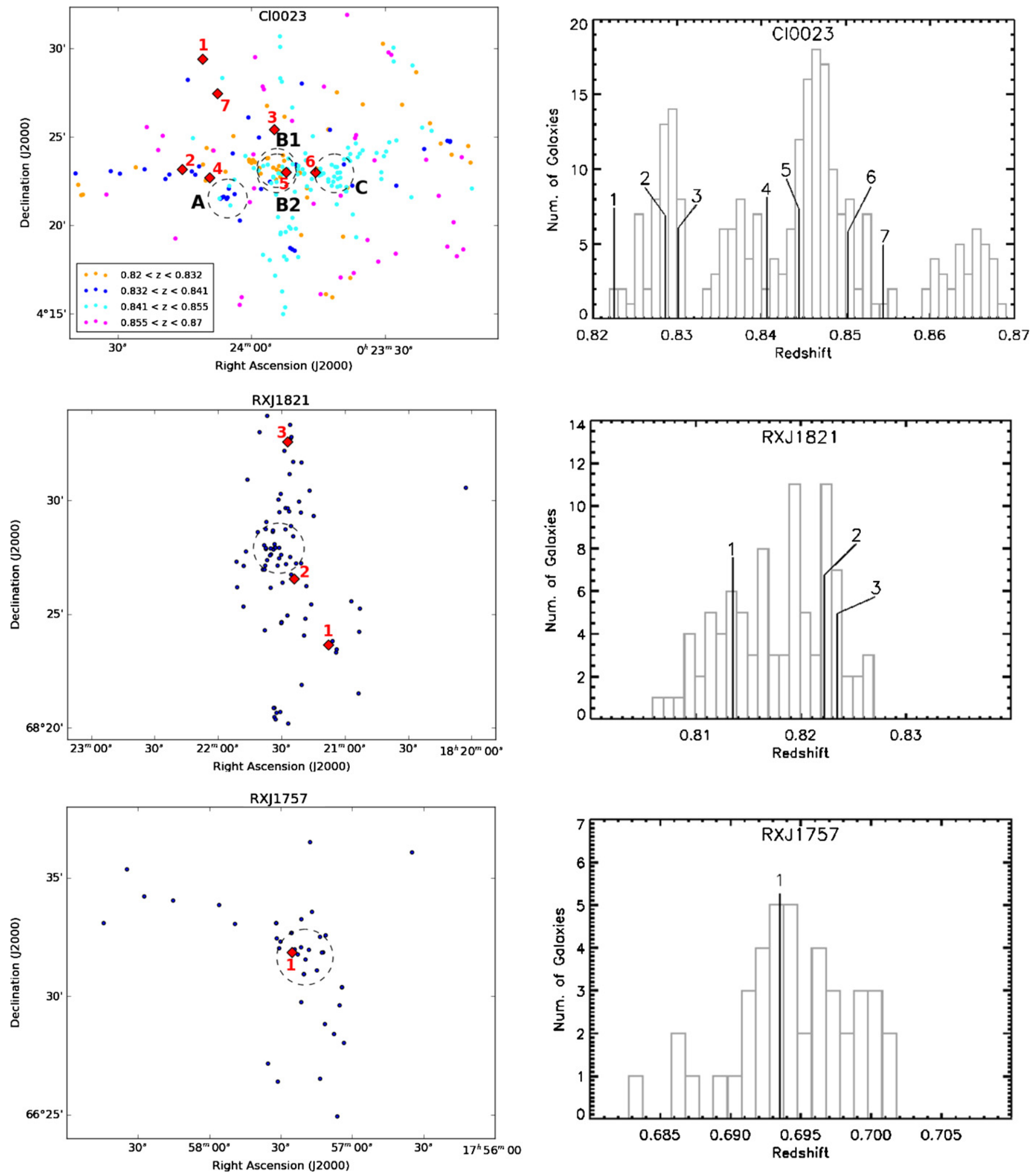

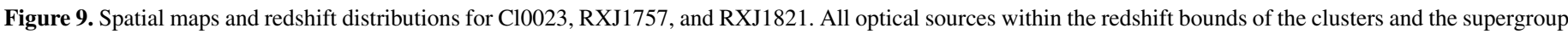

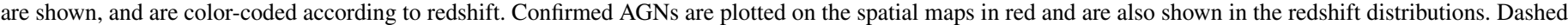
circles show the clusters and groups in the structures and have radii of $0.5 h_{70}^{-1} \mathrm{Mpc}$ at their respective redshifts.

Morphological analysis by Kocevski et al. (2009a) has shown that $\sim 67 \%$ of the X-ray AGNs in Cl1604 have had recent mergers or tidal interactions, which could fuel star formation through starburst events. More recent mergers or interactions are one possible explanation for some of the color differences that we see between the AGN host galaxies in the evolved and unevolved structures. In particular, we find that nine of the AGN host galaxies are members of a kinematic close pair with a relative line-of-sight velocity of $\Delta v \leqslant 350 \mathrm{~km} \mathrm{~s}^{-1}$ and projected physical separation (on the plane of the sky) of $\Delta r_{p} \leqslant 70 h_{70}^{-1} \mathrm{kpc}$ (e.g., Lin et al. 2007). Two are in C10023, five in Cl1604, one in Cl1324, and one in RXJ1821 (see Table 8). Those AGN hosts 
Table 8

AGN Summary

\begin{tabular}{|c|c|c|c|c|c|c|c|c|c|c|}
\hline Structure & Number & $\begin{array}{c}\text { R.A. } \\
\text { (J2000) }\end{array}$ & $\begin{array}{c}\text { Decl. } \\
(\mathrm{J} 2000)\end{array}$ & $z$ & Soft & $\begin{array}{c}L_{x}^{\mathrm{a}} \\
\text { Hard }\end{array}$ & Full & $\begin{array}{l}\text { Det. } \\
\text { Sig. }\end{array}$ & $\begin{array}{c}\text { Nearest } \\
\text { Clus. }(\mathrm{Mpc})^{\mathrm{c}}\end{array}$ & $\begin{array}{c}\text { RS } \\
\text { Offset }^{\mathrm{d}}\end{array}$ \\
\hline $\mathrm{Cl0023}$ & 1 & 002410.9 & +042923 & 0.823 & 5.6 & 46.7 & 52.3 & 12.2 & $\ldots$ & -1.86 \\
\hline $\mathrm{Cl} 10023$ & 2 & 002415.5 & +042309 & 0.829 & 7.8 & e & 7.8 & 10.1 & 1.38 & 0.67 \\
\hline $\mathrm{Cl} 0023$ & $3^{f}$ & 002354.9 & +042524 & 0.830 & 0.5 & 5.1 & 5.6 & 2.6 & 1.00 & 0.33 \\
\hline $\mathrm{Cl} 0023$ & 4 & 002409.4 & +042241 & 0.841 & 24.3 & 35.8 & 60.1 & 39.9 & 0.71 & -6.08 \\
\hline $\mathrm{Cl} 10023$ & 5 & 002352.2 & +042259 & 0.844 & 24.8 & 83.8 & 108.6 & 63.7 & 0.25 & -2.85 \\
\hline $\mathrm{Cl} 10023$ & $6^{\mathrm{f}}$ & 002345.6 & +042259 & 0.850 & 10.8 & 17.0 & 27.8 & 23.2 & 0.48 & -4.70 \\
\hline $\mathrm{Cl} 0023$ & 7 & 002407.6 & +042726 & 0.854 & 3.1 & 3.4 & 6.5 & 3.9 & $2.45^{*}$ & -1.72 \\
\hline Cl1604 & $1^{\mathrm{f}}$ & 160423.9 & +431126 & 0.867 & 14.5 & 22.3 & 36.7 & 27.8 & 1.23 & -4.43 \\
\hline Cl1604 & $2^{f}$ & 160425.9 & +431245 & 0.871 & 5.5 & 8.7 & 14.2 & 10.4 & 0.66 & -1.48 \\
\hline Cl1604 & $3^{\mathrm{f}, \mathrm{g}}$ & 160415.6 & +431016 & 0.900 & 17.0 & 31.4 & 48.3 & 31.2 & $1.90^{*}$ & -1.84 \\
\hline Cl1604 & 4 & 160437.6 & +430858 & 0.900 & 0.9 & 4.4 & 5.3 & 2.4 & 2.44 & $\ldots$ \\
\hline $\mathrm{Cl} 1604$ & 5 & 160406.1 & +431807 & 0.913 & 18.3 & 29.1 & 47.4 & 19.3 & $1.08^{*}$ & -1.02 \\
\hline $\mathrm{Cl1604}$ & $6^{\mathrm{f}}$ & 160436.7 & +432141 & 0.923 & 6.3 & 18.1 & 24.4 & 9.8 & 0.36 & -3.64 \\
\hline Cl1604 & 7 & 160401.3 & +431351 & 0.927 & 12.3 & 25.0 & 37.3 & 18.6 & 1.03 & $\ldots$ \\
\hline $\mathrm{Cl} 1604$ & 8 & 160405.1 & +431519 & 0.934 & 3.6 & 4.7 & 8.4 & 4.3 & 0.29 & -1.07 \\
\hline Cl1604 & 9 & 160410.9 & +432111 & 0.935 & 0.9 & 10.7 & 11.6 & 4.0 & $1.87^{*}$ & 0.97 \\
\hline Cl1604 & $10^{\mathrm{f}}$ & 160408.2 & +431736 & 0.937 & 5.0 & 24.9 & 30.0 & 7.1 & 0.84 & -2.47 \\
\hline Cl1324 & $1^{\mathrm{f}}$ & 132451.4 & +301239 & 0.660 & 1.6 & 0.7 & 2.3 & 4.1 & $0.44^{*}$ & -2.85 \\
\hline Cl1324 & 2 & 132436.4 & +302316 & 0.662 & 1.0 & 6.8 & 7.8 & 4.2 & $\ldots$ & -2.66 \\
\hline Cl1324 & 3 & 132504.5 & +302207 & 0.696 & 1.2 & 0.6 & 1.8 & 2.1 & $\ldots$ & -2.53 \\
\hline $\mathrm{Cl} 1324$ & 4 & 132452.9 & +305218 & 0.697 & 1.4 & $\mathrm{e}$ & 1.4 & 3.3 & 2.6 & -1.79 \\
\hline Cl1324 & 5 & 132452.0 & +305051 & 0.700 & e & 10.9 & 10.9 & 6.2 & $\ldots$ & -1.23 \\
\hline Cl1324 & 6 & 132428.8 & +305320 & 0.778 & 1.6 & 0.8 & 2.4 & 3.0 & $2.9^{*}$ & -0.24 \\
\hline RXJ1821 & 1 & 182107.7 & +682338 & 0.813 & 1.9 & 2.5 & 4.4 & 2.7 & 2.17 & -0.51 \\
\hline RXJ1821 & $2^{f}$ & 182123.9 & +68 2633 & 0.822 & 3.4 & 4.1 & 7.5 & 5.5 & 0.67 & -1.20 \\
\hline RXJ1821 & 3 & 182127.0 & +683234 & 0.824 & 8.6 & 10.6 & 19.3 & 10.3 & 2.13 & -0.66 \\
\hline RXJ1757 & 1 & 175725.2 & +663150 & 0.693 & 1.9 & 5.1 & 7.1 & 6.1 & 0.24 & -2.11 \\
\hline
\end{tabular}

Notes.

${ }^{a}$ Rest-frame X-ray luminosity in units of $10^{42} \mathrm{erg} \mathrm{s}^{-1}$. Soft, hard, and full bands are defined as $0.5-2.0,2.0-8.0$, and $0.5-8.0 \mathrm{keV}$, respectively.

${ }^{\mathrm{b}}$ Largest detection significance (in $\sigma$ 's) in the three X-ray bands.

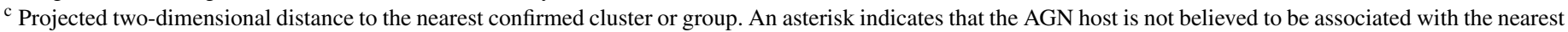

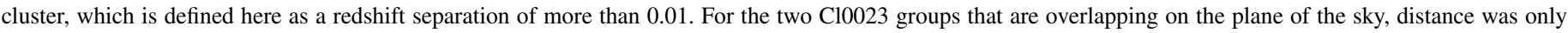

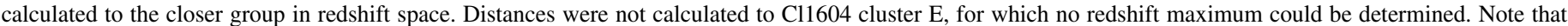
only Clusters A, B, D, and I are confirmed in C11324. Only distances less than $3 \mathrm{Mpc}$ are listed.

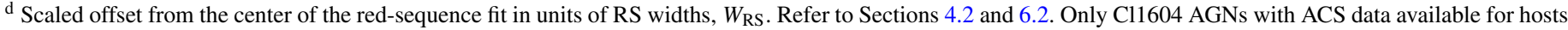
are included.

' Undetected in the respective band.

${ }^{\mathrm{f}}$ Member of a close kinematic pair (see Section 6.2).

g Has two close kinematically associated companions.

in pairs include three of the four galaxies with the largest redsequence offsets (i.e., the bluest), all of which are members of the unevolved structures. Based on their $z^{\prime}$ magnitudes or measured stellar masses (in the case of the Cl1604 members; see Lemaux et al. 2011), seven out of the nine kinematic pairs have flux or mass ratios of $\gtrsim 50 \%$, implying a major merger scenario.

The differences in color and, perhaps, merger activity are likely related to the increased level of star formation and starburst activity in the unevolved compared to evolved structures (see Section 4.3). To explore the connection between the AGN and star formation history, we can use our high-resolution spectroscopy to examine the average spectral properties of their host galaxies.

\subsection{Average Spectral Properties of AGN Hosts}

We measure the average spectral properties of the AGN host galaxies in the five ORELSE structures using three composite spectra: one comprised of six AGNs from $\mathrm{Cl} 0023,{ }^{14}$ one

\footnotetext{
14 While we have seven AGNs in our Cl0023 sample, one was not included in
} the composite spectrum. Our Cl0023 sample contains two AGNs which are not comprised of all ten AGNs from Cl1604, and one comprised of the ten AGNs from the combined fields of Cl1324, RXJ1821, and RXJ1757. The last spectrum combines all the evolved structures, necessitated by the low number of AGNs in RXJ1821 and RXJ1757 compared with the other structures. These three composite spectra are shown in Figure 11, and measurements of spectral features are listed in Table 9.

First, we can see that the AGN hosts in all three groupings have substantial [O II] emission. In Cl1604 and the evolved structures, most of this emission is probably from the AGNs, rather than star formation. Six of the ten AGN host galaxies in our Cl1604 sample were analyzed by Kocevski et al. (2011a) using the Keck II Near-infrared Echelle Spectrograph (NIRSPEC; McLean et al. 1998). For five out of the six targets, Kocevski et al. (2011a) found that the $\left[\mathrm{N}_{\mathrm{II}}\right] / \mathrm{H} \alpha$ flux ratio was too large for a normal star-forming galaxy, which implies that AGNs

type-2 (Cl0023 is the only structure with any such AGN). One of these AGNs has very broad-line features (rotational velocities $\gtrsim 1000 \mathrm{~km} \mathrm{~s}^{-1}$ ) which would dominate a composite spectrum and was therefore not included. While the other has a very broad Ne v line, the other features are narrow and do not dominate the composite spectrum. As a result, it was included. 
Table 9

Spectral Measurements of AGN Hosts

\begin{tabular}{|c|c|c|c|c|c|}
\hline Bin & $\begin{array}{c}\text { Number of } \\
\text { Galaxies }\end{array}$ & $\begin{array}{c}\mathrm{EW}([\mathrm{O} \text { II }])^{\mathrm{a}} \\
(\AA)\end{array}$ & $\begin{array}{c}\mathrm{EW}(\mathrm{H} \delta)^{\mathrm{a}, \mathrm{b}} \\
(\AA)\end{array}$ & $D_{\mathrm{n}}(4000)^{\mathrm{c}}$ & $\mathrm{Ca}$ II $\mathrm{H}+\mathrm{H} \epsilon / \mathrm{Ca}$ II $\mathrm{K}^{\mathrm{d}}$ \\
\hline $\mathrm{Cl} 0023$ & 6 & $-11.20 \pm 0.21$ & $0.36 \pm 0.39$ & $1.05 \pm 0.003$ & $0.42 \pm 0.07$ \\
\hline Cl1604 & 10 & $-7.09 \pm 0.17$ & $2.14 \pm 0.19$ & $1.22 \pm 0.005$ & $1.58 \pm 0.13$ \\
\hline Evolved Sys. ${ }^{\mathrm{e}}$ & 10 & $-12.92 \pm 0.21$ & $4.51 \pm 0.15$ & $1.44 \pm 0.006$ & $1.02 \pm 0.02$ \\
\hline
\end{tabular}

Notes.

${ }^{a}$ Measured using bandpasses from Fisher et al. (1998).

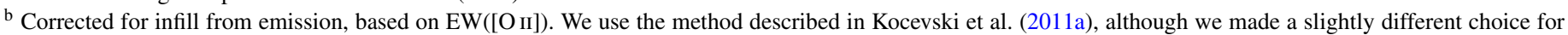
the relationship between $\mathrm{EW}(\mathrm{H} \alpha)$ and $\mathrm{EW}([\mathrm{O} \mathrm{II}])$ appropriate for Seyfert galaxies.

${ }^{c}$ Measured using bandpasses from Balogh et al. (1999).

$\mathrm{d}$ The equivalent widths are measured by fitting a line to the background and a Gaussian to the absorption feature.

e Includes C11324, RXJ1821, and RXJ1757.

are the dominant contributor to [O II] emission (Kauffmann et al. 2003). The Cl1324, RXJ1821, and RXJ1757 structures have AGNs mostly near or on the red sequence. In addition, we found in Section 4 that star formation is low in all three structures ( $\lesssim 30 \%$ of all galaxies are star forming). Because of this low star formation rate, and because Lemaux et al. (2010) found that $\sim 90 \%$ of the red $[\mathrm{O} \mathrm{II}]$ emitters are dominated by LINER/Seyfert emission, it is likely that most of the [O II] emission from the AGN host galaxies in the evolved structures comes from the AGNs as well.

Deciphering the origin of the $[\mathrm{O} \mathrm{II}]$ emission in the AGN host galaxies in $\mathrm{ClO023}$ is not as straightforward. While some AGN host galaxies in $\mathrm{Cl} 10023$ are on the red sequence, the structure also has the bluest host galaxies in our sample. Also, as discussed in Section 4, there is significant star formation in the general population of $\mathrm{ClO023}$. While this is also true of C11604, the NIRSPEC results of Kocevski et al. (2011a) showed most of the [O II] emission in the AGN host galaxies in that structure comes from the AGNs. However, we do not have any near-IR spectroscopic data for the Cl0023 AGN, so we must use other means to determine the emission source. The average $[\mathrm{Ne} \mathrm{III}] /[\mathrm{O} \mathrm{II}]$ ratio of the $\mathrm{Cl0023} \mathrm{X}$-ray AGN hosts is 0.429 , typical of type-2 AGN emission, emission from metalpoor star formation, or a superposition of the two processes (Nagao et al. 2006; Trouille et al. 2011). Note that Cl1604 and the evolved structures have values of the $[\mathrm{Ne} \mathrm{III}] /\left[\mathrm{O}_{\mathrm{II}}\right]$ ratio of 0.549 and 0.229 , respectively, also typical of type-2 AGN emission. Combining this result with the blue colors of the $\mathrm{Cl0023}$ hosts and the high fraction of star-forming galaxies, it is likely that the observed [O II] emission of the AGN hosts in $\mathrm{Cl0023}$ is due to a combination of normal star formation and type-2 AGN activity.

Examination of the Balmer features reveals further insight into the star formation histories of the AGN host galaxies. Specifically, based on a single stellar population model, the $\mathrm{EW}(\mathrm{H} \delta)$ rises quickly from zero after a starburst in a galaxy, peaks after about 300-500 Myr, and then declines back to approximately zero at $\sim 1$ Gyr after the burst (Poggianti \& Barbaro 1997). Infill can complicate the interpretation of this feature, so care must be taken when measuring the line strength. For the composite spectrum of the Cl0023 AGN hosts, the equivalent width of $\mathrm{H} \delta$, attempting to correct for infill, is consistent with zero. However, we observe strong emission from other Balmer features, $\operatorname{EW}(H \beta)=-7.81 \AA^{15}$ and $\operatorname{EW}(H \gamma)=$

\footnotetext{
15 Because a significant number of the AGN host spectra across all fields do
} not have spectral coverage including the $\mathrm{H} \beta$ line, Figure 11 was not drawn out
$-2.55 \AA$, suggesting that emission infill has a significant effect on the measured $\mathrm{EW}(\mathrm{H} \delta)$. This infill could be due to emission from $\mathrm{H}$ II regions, emission from AGNs or some other LINER processes, or from continuum emission produced by $\mathrm{O}$ stars. Since we observe $\mathrm{H} \beta$ and $\mathrm{H} \gamma$ in emission, it is unlikely that $\mathrm{O}$ stars are solely responsible for the observed $\mathrm{EW}(\mathrm{H} \delta)$. The Balmer emission lines observed in the Cl0023 AGN hosts are not broad ${ }^{16}$ and are quite strong. In the average type- 2 AGNs, a large fraction of the Balmer emission originates from star formation (Kauffmann et al. 2003), which suggests ongoing star formation in the $\mathrm{Cl0023}$ hosts. Compared to Cl0023, $\mathrm{H} \gamma$ emission from the $\mathrm{Cl1604}$ hosts is low, with $\mathrm{EW}(\mathrm{H} \gamma)=$ $-0.69 \pm 0.17$, which is consistent with a lower level of star formation in the supercluster. ${ }^{17}$ Balmer lines for the evolved structures are in absorption, $\mathrm{EW}(\mathrm{H} \beta)=0.48 \pm 0.12$ and $\mathrm{EW}(\mathrm{H} \gamma)=1.41 \pm 0.16$, consistent with an even lower level of star formation. These results combined with the earlier result analyzing the average $[\mathrm{Ne} \mathrm{III}] /\left[\mathrm{O}_{\mathrm{II}}\right]$ ratios of the AGN hosts strongly indicates that star formation is occurring in the $\mathrm{ClO023}$ galaxies coevally with AGN activity, while less star formation activity is occurring in the other structures.

The $\mathrm{H} \delta$ equivalent widths, combined with measurements of the $4000 \AA$ break, suggest that starbursts have occurred more recently in the average $\mathrm{Cl} 1604$ AGN host compared to those in the evolved structures. Larger values of $D_{\mathrm{n}}(4000)$ indicate a more passive galaxy, with an older average stellar population, which could mean that more time has passed since the cessation of star formation (Balogh et al. 1999; Kauffmann et al. 2003). The $\mathrm{EW}(\mathrm{H} \delta)$ measured from the $\mathrm{Cl1604}$ composite is $2.14 \pm 0.19 \AA$, roughly half of that from the evolved structures composite. Similarly, the C11604 composite has a lower value for $D_{\mathrm{n}}(4000)$ than those in the evolved structures, indicating that the average $\mathrm{Cl} 1604$ host is more actively star forming or has a younger stellar population on average. This result is supported by the bluer colors of the $\mathrm{Cl} 1604$ AGN hosts compared to those in the evolved structures. All of these results imply the average Cl1604 host has had a starburst more recently than the average AGN host in the evolved structures.

The AGN host composite spectrum for $\mathrm{Cl0023}$ has a particularly low $D_{\mathrm{n}}(4000)$ measurement (see Table 9). Since we found

\footnotetext{
to this range. However, approximately two-thirds of the AGNs in the $\mathrm{Cl} 0023$ structure do have spectral coverage for $\mathrm{H} \beta$, and the measurement presented here represents the average value for these galaxies.

16 With the exception of the type-1 AGN excluded from the composite spectrum, rotational velocities are $\lesssim 200 \mathrm{~km} \mathrm{~s}^{-1}$.

$17 \mathrm{EW}(\mathrm{H} \beta)$ could not be measured for a majority of galaxies in the $\mathrm{Cl1604}$
} host sample. 

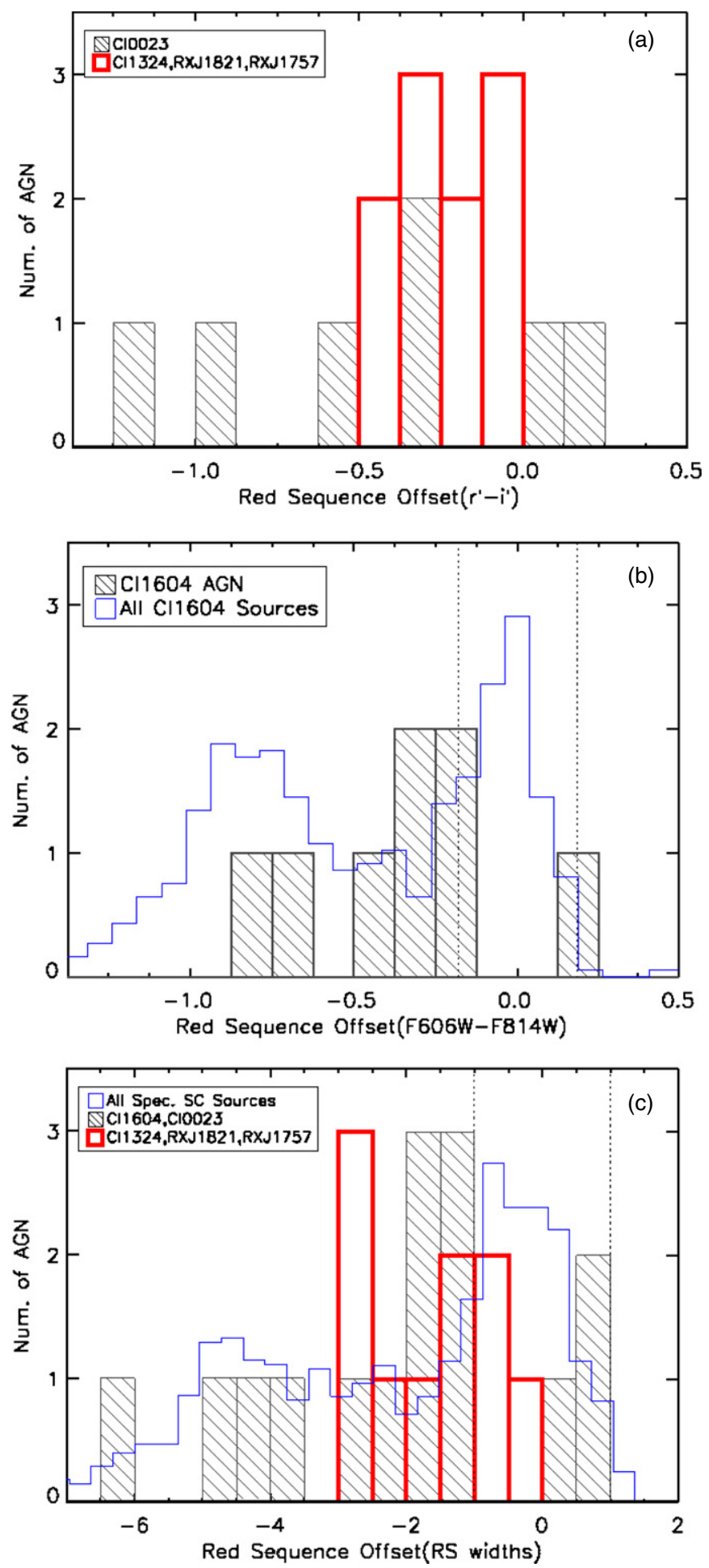

Figure 10. Offsets from the red sequence (RS) for AGN host galaxies. Panel (a) shows only those offsets calculated using the LFC $r^{\prime}-i^{\prime}$ colors, which includes all fields except for Cl1604. Panel (b) shows Cl1604 AGN host galaxies where the ACS F606W-F814W colors were used (hashed histogram). Note that these offsets are calculated from the center of the red sequence, and the RS boundaries differ from field to field. In panel (c), all five fields are included and are scaled by the red-sequence widths. In panels (b) and (c), scaled distributions of all spectroscopically confirmed supercluster members are also shown for reference (light-outlined solid histograms). In all panels, evolved structure members are indicated with thick-outlined histograms, while members of the unevolved structures are indicated by hashed histograms.

(A color version of this figure is available in the online journal.)

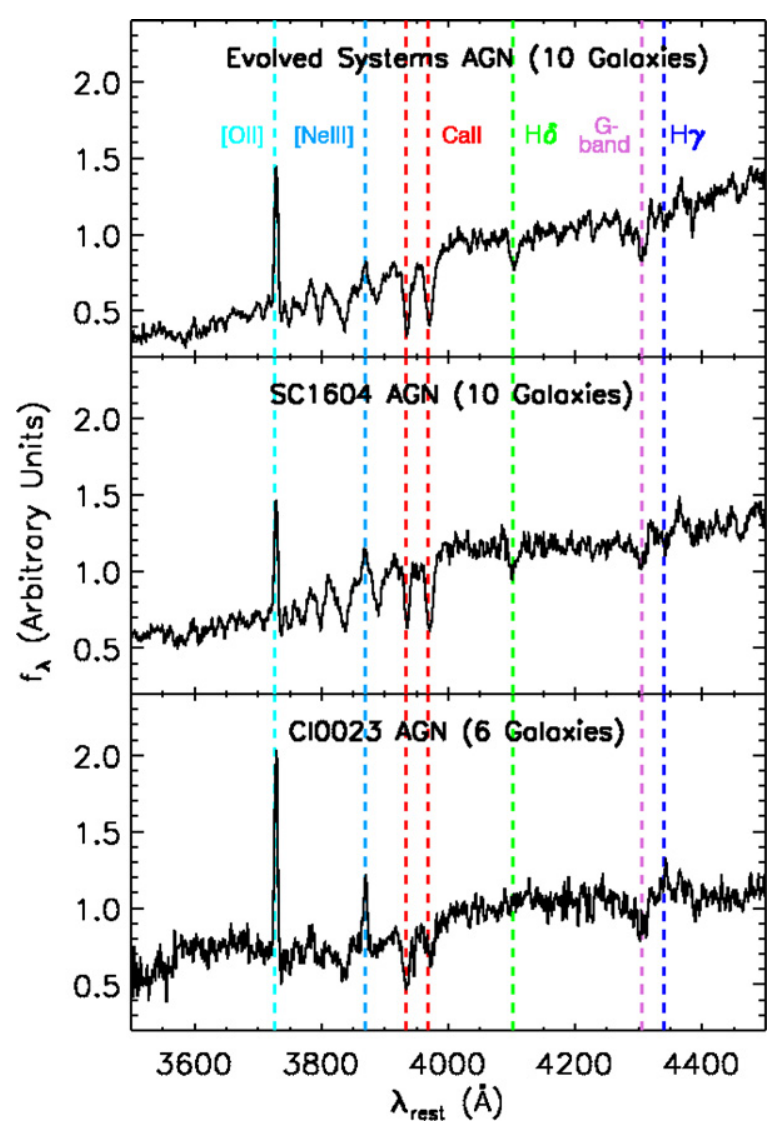

Figure 11. Composite spectra for three bins of AGNs from the five fields studied here. The top bin includes AGN hosts from Cl1324, RXJ1821, and RXJ1757. Dashed lines indicate spectral features of interest. Note the increasing depth of the $\mathrm{H} \delta$ absorption feature, which tracks recent star formation, going from the least evolved structure in our survey, $\mathrm{Cl} 10023$, to the top bin, made up of the most evolved structures. While all three spectra have significant differences, $\mathrm{Cl} 0023$ seems to be the only structure with substantial Balmer line emission and ongoing star formation.

(A color version of this figure is available in the online journal.)

that this composite spectrum (which excluded one broad-line source) was consistent with type-2 AGNs, the AGNs themselves should not contribute most of the blue continuum. This points to a stellar source, particularly O and B stars. We would then expect significant star formation in the Cl0023 hosts within the last 10-100 Myr, as indicated by the other spectral features as well.

Related to the $D_{\mathrm{n}}(4000)$ measurement, the Ca II $\mathrm{H}+\mathrm{H} \epsilon$ and the Ca II $\mathrm{K}$ lines also provide information on star formation. For $\mathrm{F}, \mathrm{G}$, and $\mathrm{K}$ stars, the ratio of these lines is constant, while the Ca II $\mathrm{H}+\mathrm{H} \epsilon / \mathrm{Ca}$ II $\mathrm{K}$ ratio increases for $\mathrm{A}$ and $\mathrm{B}$ stars as the overall $\mathrm{Ca}$ II strength decreases and the $\mathrm{H} \epsilon$ strength increases (Rose 1985). The Ca II $\mathrm{H}+\mathrm{H} \epsilon / \mathrm{Ca}$ II $\mathrm{K}$ ratio is $1.58 \pm$ 0.13 for $\mathrm{Cl} 1604$ and $1.02 \pm 0.02$ for the evolved structures (see Table 9), consistent with the evolved structures having (on average) older stellar populations. We do see a decrease in the overall strengths of both Ca II lines in the average spectrum of the Cl0023 host galaxies relative to the other structures; however, we actually measure a dramatic decrease in the ratio for Cl0023 $(0.42 \pm 0.07)$, the opposite of what is expected from a population of A and B stars. The most likely explanation is significant $\mathrm{H} \epsilon$ emission, which would be in concert with the other observed Balmer emission. The $\mathrm{H} \epsilon$ emission could be coming from some combination of AGNs 

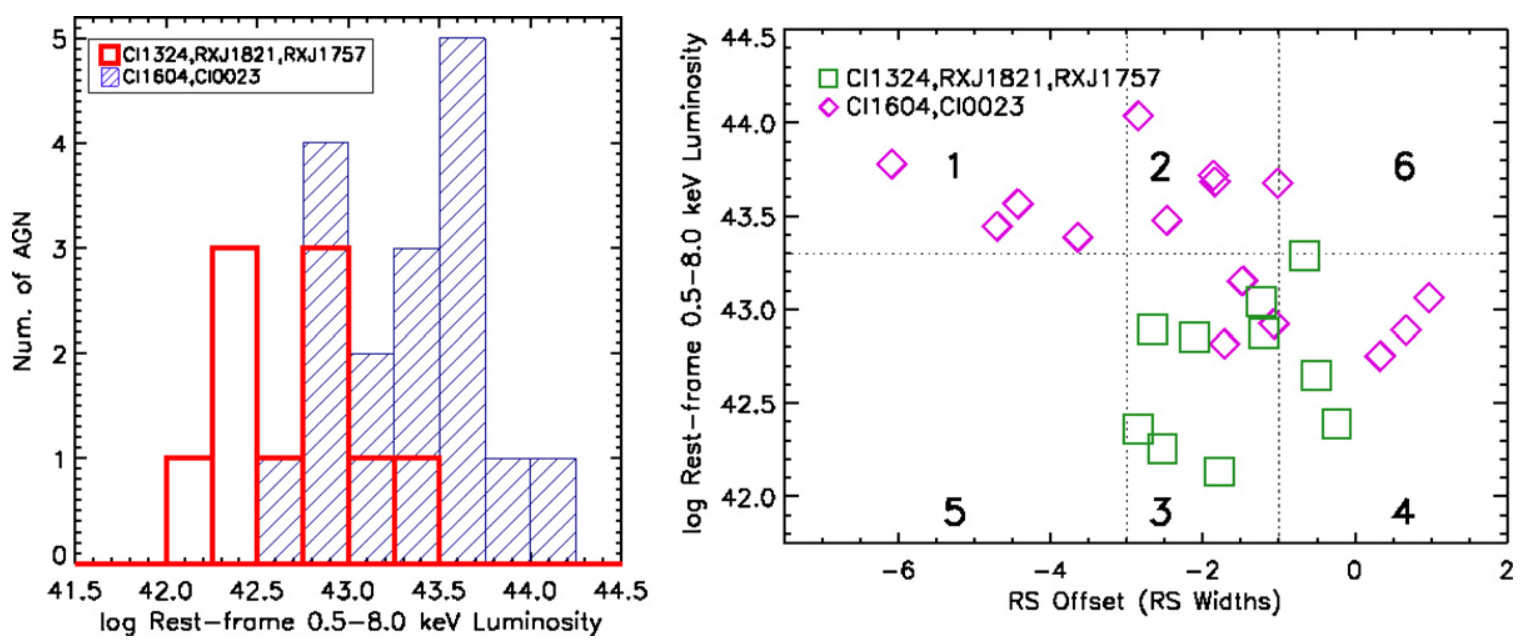

Figure 12. Left: rest-frame X-ray luminosities of AGNs in the full band ( $0.5-8 \mathrm{keV})$. Thick-outlined histograms indicate members of C11324, RXJ1821, and RXJ1757, while members of $\mathrm{Cl} 1604$ and $\mathrm{Cl} 0023$ are shown with hashed histograms. The former make up a bin of more evolved structures, and the distribution demonstrates that AGNs in these clusters tend to have lower luminosities than the less evolved structures. Right: AGN rest-frame full-band X-ray luminosities plotted against host galaxy color offsets from the red sequence, scaled by the red-sequence width. AGN hosts from $\mathrm{Cl} 1604$ and Cl0023 are shown with diamonds, while those from the three evolved structures are shown with squares. The horizontal line delineates between regions of high and low X-ray luminosity $\left(\right.$ at $L_{x}=10^{43.3}$ erg s $\left.{ }^{-1}\right)$, and the vertical lines delineate the color regions of the blue cloud, green valley, and red sequence, respectively. We observe AGNs in Regions 1-4 but none in Regions 5-6. See Section 6.4.2 for details.

(A color version of this figure is available in the online journal.)

and HiI regions, which would support previous conclusions about the level of activity in the $\mathrm{Cl0023}$ hosts.

Altogether, the composite spectra of the AGN hosts in all three bins of Figure 11 show that the average host galaxy has ongoing star formation or has had star formation within the last $\sim 1$ Gyr. However, the hosts in Cl1604 and the evolved structures each have, on average, less ongoing star formation than $\mathrm{Cl} 0023$, as evidenced by larger values of $D_{\mathrm{n}}(4000)$ and the absence of the Balmer emission that is observed in C10023. These differences suggest a progression in the temporal proximity of the last starburst event, with the hosts in Cl0023 having significant ongoing star formation characteristic of a current starburst, to those in $\mathrm{Cl} 1604$ and the evolved structures each having successively more time since the last significant starburst event. We have confirmed that none of our results based on the composite spectra are changed by removing the four lowest significance $(<3 \sigma) \mathrm{X}$-ray sources from our sample, with most spectral measurements remaining the same within the errors.

With our analysis of the composite spectra of AGN host galaxies in the different structures, we can compare the average properties of these hosts with the average properties of all spectroscopically confirmed galaxies within the same structures. In Section 4, we found that the galaxy populations in the evolved structures were largely quiescent, with little or no contribution from starburst or post-starburst galaxies. In contrast, the populations in the unevolved structures were comprised of large fractions of star-forming galaxies, with a more significant contribution from starburst or post-starburst galaxies. When comparing these results to the average spectral properties of the AGN hosts, we find that, in all cases, the average AGN host galaxy has a younger stellar population than the average galaxy in the parent structure, irregardless of the evolved or unevolved classification. This result holds even when comparing to member galaxies outside the dense cluster cores $(>0.5 \mathrm{Mpc})$, where the vast majority of X-ray AGNs reside.

The most prominent difference comes from the evolved structures where their average AGN host galaxy has significantly larger $\mathrm{EW}(\mathrm{H} \delta)$ and smaller $D_{\mathrm{n}}(4000)$ than the average structure member, indicative of a post-starburst galaxy with a substantial star formation event within the last $\sim 1$ Gyr. Such galaxies make a small contribution to the overall population in the evolved structures, which is largely quiescent. While we do observe clear differences between the spectra of the AGN hosts, with those in Cl0023 having significant ongoing star formation to those in the evolved structures having the most time since the last significant starburst event, these differences are not nearly as pronounced as the differences between the average galaxy in each structure. This suggests that AGN activity has a common origin associated with current or recent star formation.

\subsection{X-Ray Luminosity}

In this section, we explore the differences between the structures and AGN host properties based on the X-ray luminosities of the confirmed AGNs. We calculate rest-frame luminosities for $\mathrm{X}$-ray point sources with known redshifts. $K$-corrections were carried out using the power-law spectral models for sources, with a photon index of $\gamma=1.4$, described in Section 3. Luminosities are measured in the X-ray soft, hard, and full bands. A histogram of full-band rest-frame luminosity, ${ }^{18}$ binned by evolved and unevolved structures, is shown in Figure 12. The luminosity distributions in the soft and hard bands are similar to the one shown.

In the left panel of Figure 12, we can see that the AGNs in the unevolved structures have higher X-ray luminosities than those in the more evolved structures. K-S tests show that the distributions of the two bins are different at the $99 \%$ level in each of the three bands. This statistically significant result is independent of our inclusion of the four low-significance $(<3 \sigma)$ $\mathrm{X}$-ray sources.

While 10 out of 17 AGNs in the unevolved structures have full band luminosities above $L_{x}=10^{43.3} h_{70}^{-2} \mathrm{erg} \mathrm{s}^{-1}$, there are no AGNs above this limit in Cl1324, RXJ1757, or RXJ1821. We follow a Bayesian approach, using Poisson

\footnotetext{
18 Note that we are using all ten AGNs from Cl1604 here, not just the eight in
} our ACS pointings. 
statistics to calculate the likelihoods, to estimate the probability of finding no high $L_{x}$ AGNs in the evolved structures $\left(N_{\mathrm{det}}^{\mathrm{E}}=0\right)$ given the detection rate in the unevolved structures. Specifically, we calculate $P\left(N_{\text {det }}^{\mathrm{E}}=0 \mid N_{\text {det }}^{\mathrm{UE}}, N_{\text {targ }}^{\mathrm{UE}}, N_{\text {targ }}^{\mathrm{E}}\right)$. Here, $N_{\text {det }}^{\mathrm{UE}}$ is the number of high $L_{x}$ AGNs that are spectroscopically confirmed members in the unevolved structures, and $N_{\text {targ }}^{\mathrm{UE}}$ and $N_{\text {targ }}^{\mathrm{E}}$ are the total number of high $L_{x}$ AGNs that were targeted for spectroscopy in the unevolved and evolved structures, respectively. Based on this calculation, the probability of finding no high $L_{x}$ AGNs in the evolved structures is only $0.25 \%$. This result is likely related to the smaller fractions of blue cloud galaxies (and overall more quiescent populations) in Cl1324, RXJ1757, or RXJ1821 and, thus, the unavailability of large gas reservoirs.

At the faint end, there are four sources in the evolved structures below $10^{42.5} h_{70}^{-2}$ erg s${ }^{-1}$, all of which are members of C11324. However, the X-ray source counts, in all five fields, are significantly incomplete at these luminosity levels with only $\sim 5 \%$ of optically matched X-ray sources below this limit, most of which are $<3 \sigma$ detections. Therefore, we cannot say anything definitive about the lack of faint sources in the unevolved structures; however, if we remove the four low-luminosity sources, the difference between the luminosity distributions in the evolved and unevolved samples is still significant at a $95 \%$ level according to the K-S test. The reasonably high significance is clearly due to the lack of high-luminosity sources in the evolved structures.

\subsubsection{Relation to Host Galaxy Color}

In the right panel of Figure 12, we plot the full-band restframe luminosity versus the red-sequence offset, scaled by RS width (see Section 6.2). From this figure, we can see that all AGNs with host galaxies on the red sequence have lower X-ray luminosities, all with $L_{x}<10^{43.3} \mathrm{erg} \mathrm{s}^{-1}$ (Region 4). This result is not unexpected since AGN activity should diminish as the host galaxy moves onto the red sequence. We can also see that $60 \%$ of the confirmed AGNs lie in the green valley $\left(-3 W_{\mathrm{RS}}<\Delta C<-W_{\mathrm{RS}}\right.$; Regions 2 and 3). Most interestingly, in the green valley there are almost two orders of magnitude variation in X-ray luminosity, with the unevolved structures having all of the highest $L_{X}$ sources. We try to decipher the origin of these variations in Section 6.4.2.

We also do not detect any low $L_{x}$ blue AGN (Region 5) or any high $L_{x}$ red AGN (Region 6), not necessarily unexpected given their expected gas contents. However, it is difficult to say for certain if these null results are significant. We do sample optically matched X-ray sources in these regions. Specifically, in the five fields, there are a total of 85 sources (at $>3 \sigma$ ) in Region 5 $\left(-6.1 W_{\mathrm{RS}}<\Delta C<-3 W_{\mathrm{RS}}\right.$ and $\left.10^{42.5}<L_{x}<10^{43.3} \mathrm{erg} \mathrm{s}^{-1}\right)$, of which we have targeted $24(28 \%)$. Here, we estimate the $\mathrm{X}$-ray luminosities by assuming that all sources in a particular field are at the mean redshift of the structure, and we choose the lower limit of $L_{x}=10^{42.5} \mathrm{erg} \mathrm{s}^{-1}$ so as not to be adversely affected by incompleteness (see above). Based on Barger et al. (2005), the X-ray luminosity function of field AGNs at similar redshifts in "optically normal" galaxies (comparable to the vast majority of our AGN hosts) shows increasing number densities down to $L_{x} \approx 10^{42} \mathrm{erg} \mathrm{s}^{-1}$. As a result, we would naively expect to detect a larger number of fainter X-ray sources in the blue cloud. Using the Bayesian approach described in Section 6.4, the probability of finding no AGN in Region 5 is $3 \%$, given our success rate of confirming cluster members for the high $L_{x}$ blue galaxies (Region 1). This formal probability

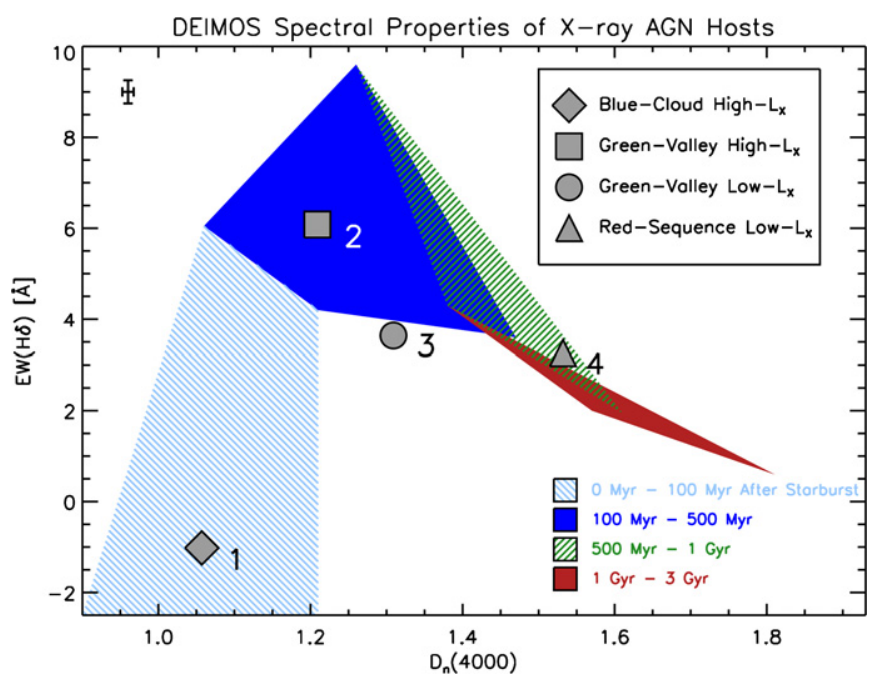

Figure 13. $\mathrm{EW}(\mathrm{H} \delta)$ vs. $D_{\mathrm{n}}(4000)$ from the composites made by AGN host galaxies in Regions 1-4 of Figure 12. For comparison, the ranges of $\mathrm{EW}(\mathrm{H} \delta)-D_{\mathrm{n}}(4000)$ phase space spanned by four different Bruzual \& Charlot (Bruzual 2007) models for various times after the starburst are shown. Because Bruzual \& Charlot models only incorporate stellar light, emission infill corrections were made for all $\mathrm{EW}(\mathrm{H} \delta)$ measurements (see Section 4.3 for details). The average host galaxy in each region either is in the process of having a starburst or has had one within the last $\lesssim 1$ Gyr. The hosts of AGNs with higher X-ray luminosities, even when comparing green-valley galaxies, have younger stellar populations, with shorter times since the last burst. A typical error bar is shown in the upper left corner.

may, in fact, be an upper limit as we would expect a higher success rate given the larger number densities at fainter X-ray luminosities. The fact that we observe no low $L_{x}$ blue galaxies in our sample may suggest that, in high-density environments compared to the field, either (1) the time to reach the highest $\mathrm{X}$-ray luminosities is shorter after AGN turn-on or (2) the host galaxies are transformed more quickly, moving to redder colors by the time their X-ray luminosities drop to lower levels.

Similarly, there are 25 sources in Region $6\left(-1 W_{\mathrm{RS}}>\Delta C>\right.$ $2 W_{\mathrm{RS}}$ and $L_{x}>10^{43.3} \mathrm{erg} \mathrm{s}^{-1}$ ), of which we have targeted $7(28 \%)$. If we assume our success rate for confirming cluster members as measured from all high $L_{x}$ AGNs (Regions 1 and 2), the probability of finding no AGN in Region 6 is $18 \%$. Although not a significant result, the conclusions for this region are, of course, more obvious as we do not expect any true red-sequence (i.e., non-dust reddened) galaxies to have enough cold gas to fuel a luminous AGN.

\subsubsection{Relation to Average Spectral Properties}

To explore the origin of the variations observed in Figure 12, we examine the average spectral properties of the host galaxies within the four distinct regions. Specifically, the sample is split by X-ray luminosity at $L_{x}=10^{43.3}$ and red-sequence offset, scaled by RS width, to delineate regions containing high $L_{x}$ blue cloud (Region 1), high $L_{x}$ (Region 2 ) and low $L_{x}$ (Region 3 ) green valley, and low $L_{x}$ red-sequence (Region 4 ) host galaxies. In Figure 13, we plot the measured $\operatorname{EW}(\mathrm{H} \delta)$ versus $D_{\mathrm{n}}(4000)$ from the spectral composites in the four regions. For comparison, we also plot post-starburst temporal regimes derived from four Bruzual (2007) models, described in Section 4.3.

The small $D_{\mathrm{n}}(4000)$ and $\mathrm{H} \delta$ in emission indicate that the high $L_{x}$ blue hosts are coeval with the starburst or ongoing star formation. As we examine galaxies in Regions 2 to 4 going from the high to low $L_{x}$ hosts in the green valley to the low $L_{x}$ hosts in the red sequence, the time since the burst gets 
progressively larger. While there is some degeneracy between time since burst and burst strength, it is clear that the low $L_{x}$ green-valley hosts are either (1) further along since the burst than their high $L_{x}$ counterparts or (2) had a weaker initial burst which could explain their lower X-ray luminosities as less gas would likely be funneled to the center. Our results are robust to removing the four lowest significance $(<3 \sigma)$ X-ray sources, as well as the four X-ray sources below $L_{x}=10^{42.5} \mathrm{erg} \mathrm{s}^{-1}$, where in both cases we are highly incomplete.

The most striking results from this spectral analysis are, first, that the average AGN host in every region is either in the process of having a starburst or has had one within last $\lesssim 1$ Gyr. This global finding clearly demonstrates the close connection between starburst and AGN activity as normal star formation does not typically produce the $\mathrm{H} \delta$ values seen in these host galaxies. Second, we do not detect high X-ray luminosity, young (as indicated by time since starburst) galaxies in the evolved structures. This result implies that the entire galaxy population in these structures (certainly in the isolated, X-rayselected ones) are more advanced, suggesting that the peak of gas consumption, seen in both star formation and AGN activity, occurred at an earlier time.

\section{DISCUSSION}

We studied AGN activity in five high-redshift clusters and superclusters in the redshift range $z \approx 0.7-0.9$. Before identifying individual AGNs, we analyzed the structures using the statistical measure of cumulative source counts. We found every structure to have X-ray excesses of $0.5 \sigma-1.5 \sigma$ with respect to the CDFN and CDFS control fields. The method is highly dependent on the field used as a sky estimate, which makes comparing results between studies difficult. Cappelluti et al. (2005) measured cumulative source counts in a number of structures in the range $0.2 \lesssim z \lesssim 1.4$ and found a dependence on redshift. While our data are consistent within the errors with the results of $\mathrm{C} 05$, our redshift range is not large enough to evaluate the relation. We note that it is difficult to use these overdensities to interpret the actual AGN activity in an individual structure, even with extensive spectroscopy, as we have attempted to do here with our large spectroscopic sample. We recommend caution in using this technique, as its precision can easily be overestimated.

We employed a maximum likelihood technique to match $\mathrm{X}$-ray sources to optical counterparts. With an extensive DEIMOS optical spectroscopic campaign with $\sim 6000$ targets, accurate redshifts have been obtained for 126 of these X-ray sources, allowing us to identify a total of 27 AGNs within all of the structures. These results show that significant spectroscopy is needed to confirm even small numbers of AGN members. We find that the spatial distribution of the AGN is largely consistent with previous work at lower redshift. Across all five structures, we find that AGN host galaxies tend to be located away from dense cores (within $0.5 \mathrm{Mpc}$ of a cluster or group center), with many instead located on the outskirts of clusters or poorer groups. ${ }^{19}$ Previous studies have found similar results up to $z \sim 1$ (Coldwell et al. 2002; Kauffmann et al. 2004; Gilmour et al. 2007; Kocevski et al. 2009b). These intermediate environments are thought to be conducive to galaxy-galaxy interactions because of the relatively high densities compared to the field and low velocity dispersions compared to cluster cores (Cavaliere et al. 1992). Our results would then lend support to

19 Only including groups with $\sigma>300 \mathrm{~km} \mathrm{~s}^{-1}$. these interactions as the trigger of X-ray AGN activity in the environments of LSSs. However, these may also be regions where gas availability is higher in the member galaxies, which could lead to increased AGN activity.

With optical counterparts to X-ray sources identified, we were able to analyze the color properties of AGN hosts. Our analysis showed that AGN host galaxies are overrepresented in the green valley. In C11604, only $\sim 17 \%$ of all the supercluster members with ACS data were within $2 W_{\mathrm{RS}}$ of the lower boundary of the red sequence, where $W_{\mathrm{RS}}$ is the width of the red sequence, defined in Section 4. However, five out of the eight AGN hosts in our ACS pointings were in this range. In fact, $36 \%$ of the host galaxies in all five structures lie within $W_{\mathrm{RS}}$ of the lower boundary of the red sequence and $60 \%$ are within $2 W_{\mathrm{RS}}$. Our results are supported by other studies which have found an overabundance of AGN activity in the green valley (Nandra et al. 2007; Georgakakis et al. 2008; Silverman et al. 2008; Hickox et al. 2009). Since the green valley is thought to be a transitional region for galaxies (Faber et al. 2007), these results suggest $\mathrm{X}$-ray AGNs in LSS are a transitional population between blue star-forming and red quiescent galaxies. However, we note that, while our sample is magnitude-limited, several studies using mass-selected samples have found less overrepresentation of AGN hosts in the green valley (Silverman et al. 2009b; Xue et al. 2010). Additionally, Cardamone et al. (2010) have found that $\sim 75 \%$ of the AGN hosts in the green valley are dustreddened blue cloud galaxies, although these results conflict with the recent studies of Rosario et al. (2011). Our results from preliminary SED fittings to AGN hosts suggest that the effect is not as drastic in our sample.

The five structures studied in this paper occupy a range of evolutionary states. Based on the [O II] and $\mathrm{H} \delta$ features of the composite spectra for each structure, we grouped our sample into the least evolved structures ("unevolved"), Cl1604 and $\mathrm{Cl0023}$, and the most evolved structures ("evolved"), C11324, RXJ1821, and RXJ1757. This distinction is based on the average stellar populations and the presence, or lack thereof, of current star formation. With these two categories, we sought to explore differences in AGN activity between structures with different galaxy populations. We did not find any significant differences between the five structures when examining the cumulative source counts or the spatial distributions of the AGN hosts. However, the AGN host galaxies in the unevolved structures were skewed more toward bluer colors, although this was not at a statistically significant level for this sample size.

We did, however, find significant differences between the subsets when examining the X-ray luminosities of the AGNs and the optical spectra of their hosts. We found that AGNs in the unevolved structures tend to have higher full band $(0.5-8 \mathrm{keV}) \mathrm{X}$-ray luminosities relative to those in the evolved structures at a $99 \%$ level, with all of the most luminous AGNs $\left(L_{x}>10^{43.3} \mathrm{erg} \mathrm{s}^{-1}\right)$ found in the unevolved structures. While all AGN host galaxies either have ongoing star formation or have had a starburst within the last $\lesssim 1$ Gyr, the host galaxies in the unevolved structures are distinctly younger than those in the evolved structures, with shorter times since the last starburst as indicated by smaller average $\mathrm{EW}(H \delta)$ and $D_{\mathrm{n}}(4000)$ in their composite spectra. The average $\mathrm{Cl} 0023$ host has current star formation, and the average $\mathrm{Cl} 1604$ host has had a burst within the last $\sim 100$ Myr. We do not detect any of these young, high X-ray luminosity AGNs in the evolved structures, implying that the peak of both star formation and AGN activity occurred at 
an earlier time. We note that, regardless of whether they are members of the evolved or unevolved structures, all AGN host galaxies are younger than the average galaxy in their parent population.

We also find a large (two orders of magnitude) variation in X-ray luminosity for AGNs within the green valley, while AGNs in the red sequence have consistently lower luminosities $\left(L_{x}<10^{43.3} \mathrm{erg} \mathrm{s}^{-1}\right)$. As we move from the high to low $L_{x}$ green-valley hosts to low $L_{x}$ red-sequence hosts, the time since starburst gets progressively longer. Although there is some degeneracy between burst strength and time since burst, the low $L_{x}$ green-valley hosts are either further along since the burst than their high $L_{x}$ counterparts or have had a weaker initial burst, which may explain their lower X-ray luminosities.

The higher AGN X-ray luminosities in the unevolved structures are most likely related to their bluer colors and, hence, larger reservoirs of gas that could lead to higher levels of black hole accretion and the higher X-ray luminosities. In addition, both the X-ray and spectral results can be explained if these galaxies had more recently undergone merger-induced, or other, starburst events. Specifically, simulations and observations have found that AGN activity peaks soon after maximum star formation in a starburst event $(\sim 0.1-0.25$ Gyr; Davies et al. 2007; Schawinski et al. 2007, 2009; Wild et al. 2010; Hopkins 2011). As the AGN X-ray luminosity declines after reaching its peak, the star formation rate should be declining as well. This could explain why the most X-ray luminous AGNs are in the least evolved structures. AGN host galaxies in $\mathrm{Cl} 10023$ were also found to have significant ongoing star formation, which could mean these galaxies have had the most recent merger events, where star formation is still near its peak.

The X-ray luminosity differences between the AGNs in the evolved and unevolved structures could also be viewed as a transition from quasar mode emission toward radio mode emission, as defined in Croton et al. (2006) and McNamara \& Nulsen (2007), which is also related to the star formation rate. Since AGN feedback deters gas from falling into the core and depletes the cold gas in a galaxy, the fuel for both star formation and further AGN emission is decreased. Ultimately, the dominant fuel for black hole accretion transitions from cold gas funneled to the galactic core from the starburst event to hot halo gas, which leads to a much more quiescent state (Croton et al. 2006; McNamara \& Nulsen 2007; for a similar model, see also, e.g., Bower et al. 2006; Fanidakis et al. 2011). In our sample, the C10023 AGNs are emitting more similarly to the quasar mode, accompanied by substantial star formation. The AGN host galaxies in $\mathrm{Cl1604}$ and the evolved structures

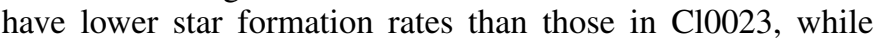
the evolved structures have AGNs with lower X-ray luminosities, which suggests that they are sequentially further along the track leading toward domination of radio mode AGN emission. If the AGNs in the evolved structures were found to be radio emitters, it would support these conclusions. Existing VLA B-array observations (at $1.4 \mathrm{GHz}$ ) of these five structures are currently being analyzed to explore this connection (C. D. Fassnacht et al. 2012, in preparation).

Altogether, many of our results could support several potential AGN triggering scenarios. Two possibilities are that (1) AGNs in these structures represent a transitional population where hosts are evolving from the blue cloud onto the red sequence or (2) AGNs represent a population evolving mainly in mass space, where red-sequence hosts have undergone episodic nuclear star formation induced by minor mergers. Additionally, many observable effects of the latter would appear similar to models in which recycled stellar material fuels central starbursts and nuclear activity in elliptical galaxies, which tend to be located on the red sequence. In the first case, AGNs are triggered by mergers or strong tidal interactions which lead to a starburst. The feedback from the AGN quenches star formation, leading to a rapid evolution across the green valley and onto the red sequence (Hopkins et al. 2005, 2007; Springel et al. 2005; Somerville et al. 2008). In the second case, red-sequence galaxies undergo minor mergers, which funnel gas into the galactic core, creating a burst of nuclear star formation and fueling the AGNs (Menanteau et al. 2001). Alternatively, recycled stellar material could create a nuclear starburst and a central instability, leading to black hole accretion (Ciotti \& Ostriker 2007). In either case, the galaxies could evolve into the green valley before AGN feedback brought them onto the red sequence again. Once on the red sequence, there would not be a large net change in color, in contrast to the dramatic color evolution of the first possibility. In the case of minor mergers, this process would mainly entail an evolution in mass.

In support of AGNs as a transitional population, many of the host galaxies across all five structures are located close to the red sequence, where the green valley should lie, although it is difficult to determine the location of the green valley in the four structures without precise ACS data. In support of all scenarios involving significant starbursts, the average AGN host in both the evolved structures and the $\mathrm{Cl1604}$ supercluster has substantial $\mathrm{H} \delta$ absorption, which is a sign of recently quenched star formation. This is expected for green-valley galaxies evolving onto the red sequence. Previous morphological analysis of part of our sample by Kocevski et al. (2009b) found that two-thirds of $\mathrm{Cl1604}$ host galaxies studied showed signs of recent or pending mergers or tidal interactions, which is expected in the context of both major and minor merger theories. In addition, we find that at least 9 of the 27 AGN host galaxies are part of a kinematic close pair. In seven of these cases, the companion galaxy has a similar stellar mass or $z^{\prime}$ magnitude to the AGN host. While being far from conclusive due to sampling and selection effects, these results could point to a major merger scenario.

Kocevski et al. (2009b) examined eight of the AGNs in Cl1604 and found that half of the hosts had blue cores in an otherwise red galaxy. Other studies have also found blue cores or blue early-type galaxies (Lee et al. 2006; Martel et al. 2007). These blue cores are predicted by minor merger simulations (Mihos \& Hernquist 1996), as well as the recycled gas models of Ciotti \& Ostriker (2007). In addition, simulations show that central black hole accretion is most highly correlated with star formation in the nucleus, as opposed to the entire galaxy (Hopkins \& Quataert 2010; Diamond-Stanic \& Rieke 2011). During a major merger, star formation peaks at later times closer to the galactic core (Hopkins 2011). This could potentially create bluer cores as well. From our data, it seems our results are ambiguous with regards to the various scenarios. However, with evidence supporting more than one possibility, our results could indicate a combination of different triggering mechanisms.

These different AGN triggering mechanisms could also potentially explain some of the differences that we see between the evolved and unevolved structures. The second AGN mode, involving episodic nuclear activity in red-sequence galaxies fueled by minor mergers, or recycled gas, is expected to involve accretion rates at a much lower Eddington ratio than in major merger-driven AGN activity (Marconi et al. 2004; Merloni \& 
Heinz 2008; Hasinger 2008). While AGN luminosity depends on both the Eddington ratio and the black hole mass (Kauffmann \& Haehnelt 2000), lower Eddington ratios will, on average, correspond to lower luminosities. A difference in Eddington ratios could potentially explain the lower X-ray luminosities that we observe in the evolved structures, if our sample of host galaxies consists of a mix of the two AGN modes. In the unevolved structures, we would expect to observe more major mergers between blue cloud galaxies due to the larger fraction of blue galaxies, fueling the brightest AGN in our sample. Since all of our structures have a substantial number of red-sequence galaxies we would expect all structures to have galaxies undergoing the second mode of AGN activity, whether induced by minor mergers or, perhaps, recycled gas in ellipticals. We would then expect the X-ray luminosity distributions of the evolved and unevolved structures to look similar except for a tail of higher luminosity objects in the unevolved structures, which is roughly what we observe. While a combination of the two AGN modes considered could explain our results, they could also be explained if major mergers were the primary driver, and the quasar mode is less dominant for the AGNs in the evolved structures, because of the larger red fraction therein. With our current data, we still lack the ability to distinguish between the different triggering mechanisms with our data. Breaking the degeneracy will require high-resolution imaging to examine the morphologies of the AGN hosts and the colors of their cores in the structures other than $\mathrm{Cl1604}$, as well as reliable stellar masses and HST data, for measuring bulge-to-disk ratios, with which we could reliably calculate Eddington ratios.

\section{CONCLUSIONS}

In summary, we find that most of the X-ray AGN hosts, across all five structures, avoid the dense cluster cores, in agreement with a number of previous studies at a range of redshifts. We interpret this to mean that X-ray AGN activity is preferentially triggered in intermediate-density environments, such as the outskirts of clusters. We also find many AGN host galaxies in or near the green valley, with $36 \%$ within one red-sequence width of the lower boundary of the red sequence and $60 \%$ within two red-sequence widths. With numerous other studies finding a similar connection, this implies that there is an association between this transitional region and AGN activity.

We divided our sample of five structures into two groups: the more and less evolved structures, which we separated using composite spectra made of all of their spectroscopically confirmed member galaxies. We define the more evolved structures as those with member galaxies that exhibit, on average, less [O II] emission and less $\mathrm{H} \delta$ absorption, where the [O II] and $\mathrm{H} \delta$ lines are taken as indicators of ongoing and recent star formation, respectively. The more evolved structures also have galaxy populations with a higher red fraction than the less evolved structures. Our spectral results indicate that the AGN hosts in the less evolved structures have more ongoing star formation, while those in the more evolved structures have stronger average $\mathrm{H} \delta$ features. Stronger $\mathrm{H} \delta$ lines are indicative of star formation within the past $\sim 1 \mathrm{Gyr}$, and our results indicate starbursts occurred more recently in the AGN host galaxies in the less evolved structures. However, all of the AGN hosts, regardless of whether they are members of the more evolved or less evolved structures, are younger than the average galaxy in their parent population.

We also found that AGNs in the less evolved structures had more luminous X-ray emission. This may be expected, since these structures contain the highest fraction of blue galaxies which are likely to have larger reservoirs of cool gas to fuel nuclear activity. If AGNs were triggered more recently in the less evolved structures, as the spectral data suggest, the difference in luminosity could also be related to a transition from "quasar mode" emission in newly triggered AGNs to "radio mode" emission at later times as supplies of inflowing cool gas are shut off.

We consider several scenarios for AGN triggering that are in agreement with our results. AGNs are triggered by major mergers or tidal interactions between blue cloud galaxies and/or AGNs are triggered episodically in red-sequence galaxies, fueled by recycled stellar material or induced by minor mergers. Each of these scenarios could explain the association of AGN hosts with the green valley. Also, the $\mathrm{H} \delta$ absorption we observe could be indicative of the quenching of star formation that drives galaxies across the green valley. A previous study of the Cl1604 AGN by Kocevski et al. (2009b) found that a majority of hosts had recent or pending mergers. Half of the hosts had blue cores in otherwise red galaxies, which could support the second two scenarios, although other explanations are possible. Some of our results, such as the difference in X-ray luminosities, could be explained if the AGNs in our sample were triggered by a mix of these modes, with a larger fraction of AGNs in the less evolved structures triggered by major mergers. However, we cannot distinguish between the triggering scenarios with our data, so the cause of the AGN activity is still ambiguous. Future work investigating AGN host morphologies, examining for blue cores, and calculating Eddington ratios could potentially break the degeneracy.

The authors thank Phil Marshall and Robert Lupton for useful conversations. This work is supported by the Chandra General Observing Program under award numbers GO6-7114X, GO7-8126X, GO8-9123A, and GO9-0139A. In addition, we acknowledge support by the National Science Foundation under grant AST-0907858. The spectrographic data presented herein were obtained at the W. M. Keck Observatory, which is operated as a scientific partnership among the California Institute of Technology, the University of California, and the National Aeronautics and Space Administration. The Observatory was made possible by the generous financial support of the W. M. Keck Foundation. As always, we thank the indigenous Hawaiian community for allowing us to be guests on their sacred mountain. We are most fortunate to be able to conduct observations from this site.

\section{REFERENCES}

Baldry, I. K., Glazebrook, K., Brinkmann, J., et al. 2004, ApJ, 600, 681 Balogh, M. L., Morris, S. L., Yee, H. K. C., Carlberg, R. G., \& Ellingson, E. 1999, ApJ, 527, 54

Barger, A. J., Cowie, L. L., Mushotzky, R. F., et al. 2005, AJ, 129, 578

Bluck, A. F. L., Conselice, C. J., Almaini, O., et al. 2011, MNRAS, 410, 1174

Bournaud, F., Chapon, D., Teyssier, R., et al. 2011, ApJ, 730, 4

Bower, R. G., Benson, A. J., Malbon, R., et al. 2006, MNRAS, 370, 645

Boyle, B. J., \& Terlevich, R. J. 1998, MNRAS, 293, L49

Brandt, W. N., Alexander, D. M., Hornschemeier, A. E., et al. 2001, AJ, 122, 2810

Bruzual, G. 2007, in ASP Conf. Ser. 374, From Stars to Galaxies: Building the Pieces to Build Up the Universe, ed. A. Vallenari, R. Tantalo, L. Portinari, \& A. Moretti (San Francisco, CA: ASP), 303

Butcher, H., \& Oemler, A., Jr. 1984, ApJ, 285, 426

Calzetti, D., Armus, L., Bohlin, R. C., et al. 2000, ApJ, 533, 682

Cappelluti, N., Cappi, M., Dadina, M., et al. 2005, A\&A, 430, 39

Cardamone, C. N., Urry, C. M., Schawinski, K., et al. 2010, ApJ, 721, L38

Cavaliere, A., Colafrancesco, S., \& Menci, N. 1992, ApJ, 392, 41

Ciotti, L., \& Ostriker, J. P. 2007, ApJ, 665, 1038

Coldwell, G. V., Martínez, H. J., \& Lambas, D. G. 2002, MNRAS, 336, 207 
Colless, M., Dalton, G., Maddox, S., et al. 2001, MNRAS, 328, 1039 Crawford, D. F., Jauncey, D. L., \& Murdoch, H. S. 1970, ApJ, 162, 405 Croton, D. J., Springel, V., White, S. D. M., et al. 2006, MNRAS, 365, 11 Davies, R. I., Müller Sánchez, F., Genzel, R., et al. 2007, ApJ, 671, 1388 Diamond-Stanic, A. M., \& Rieke, G. H. 2011, ApJ, in press (arXiv:1106.3565) Dickey, J. M., \& Lockman, F. J. 1990, ARA\&A, 28, 215

Dressler, A., Oemler, A., Jr., Poggianti, B. M., et al. 2004, ApJ, 617, 867 Dressler, A., \& Shectman, S. A. 1988, AJ, 95, 284

Dressler, A., Smail, I., Poggianti, B. M., et al. 1999, ApJS, 122, 51 Eastman, J., Martini, P., Sivakoff, G., et al. 2007, ApJ, 664, L9

Faber, S. M., Phillips, A. C., Kibrick, R. I., et al. 2003, Proc. SPIE, 4841, 1657

Faber, S. M., Willmer, C. N. A., Wolf, C., et al. 2007, ApJ, 665, 265

Fanidakis, N., Baugh, C. M., Benson, A. J., et al. 2011, MNRAS, 410, 53

Ferrarese, L., \& Merritt, D. 2000, ApJ, 539, L9

Fisher, D., Fabricant, D., Franx, M., \& van Dokkum, P. 1998, ApJ, 498, 195

Ford, H. C., Clampin, M., Hartig, G. F., et al. 2003, Proc. SPIE, 4854, 81

Fruscione, A., McDowell, J. C., Allen, G. E., et al. 2006, Proc. SPIE, 6270, 60

Gal, R. R., Lemaux, B. C., Lubin, L. M., Kocevski, D., \& Squires, G. K. 2008, ApJ, 684, 933

Gal, R. R., \& Lubin, L. M. 2004, ApJ, 607, L1

Gal, R. R., Lubin, L. M., \& Squires, G. K. 2005, AJ, 129, 1827

Gebhardt, K., Bender, R., Bower, G., et al. 2000, ApJ, 539, L13

Gehrels, N. 1986, ApJ, 303, 336

Georgakakis, A., Nandra, K., Yan, R., et al. 2008, MNRAS, 385, 2049

Georgantopoulos, I., Rovilos, E., \& Comastri, A. 2011, A\&A, 526, A46

Gilmour, R., Gray, M. E., Almaini, O., et al. 2007, MNRAS, 380, 1467

Gioia, I. M., Henry, J. P., Mullis, C. R., et al. 2003, ApJS, 149, 29

Gioia, I. M., Maccacaro, T., Schild, R. E., et al. 1990, ApJS, 72, 567

Gioia, I. M., Wolter, A., Mullis, C. R., et al. 2004, A\&A, 428, 867

Gladders, M. D., Lopez-Cruz, O., Yee, H. K. C., \& Kodama, T. 1998, ApJ, 501, 571

Gunn, J. E., Hoessel, J. G., \& Oke, J. B. 1986, ApJ, 306, 30

Häring, N., \& Rix, H.-W. 2004, ApJ, 604, L89

Hasinger, G. 2008, A\&A, 490, 905

Heckman, T. M., Kauffmann, G., Brinchmann, J., et al. 2004, ApJ, 613, 109

Henry, J. P., Mullis, C. R., Voges, W., et al. 2006, ApJS, 162, 304

Hickox, R. C., Jones, C., Forman, W. R., et al. 2009, ApJ, 696, 89

Hopkins, P. F. 2011, MNRAS, in press (arXiv:1101.4230)

Hopkins, P. F., Bundy, K., Hernquist, L., \& Ellis, R. S. 2007, ApJ, 659, 976

Hopkins, P. F., \& Hernquist, L. 2006, ApJS, 166, 1

Hopkins, P. F., Hernquist, L., Martini, P., et al. 2005, ApJ, 625, L71

Hopkins, P. F., \& Quataert, E. 2010, MNRAS, 407, 1529

Johnson, O., Best, P. N., \& Almaini, O. 2003, MNRAS, 343, 924

Kartaltepe, J. S., Sanders, D. B., Scoville, N. Z., et al. 2007, ApJS, 172, 320

Kauffmann, G., \& Haehnelt, M. 2000, MNRAS, 311, 576

Kauffmann, G., Heckman, T. M., Tremonti, C., et al. 2003, MNRAS, 346, 1055

Kauffmann, G., White, S. D. M., Heckman, T. M., et al. 2004, MNRAS, 353, 713

Kim, M., Kim, D.-W., Wilkes, B. J., et al. 2007, ApJS, 169, 401

Kocevski, D. D., Faber, S. M., Mozena, M., et al. 2011b, ApJ, in press

Kocevski, D. D., Lemaux, B. C., Lubin, L. M., et al. 2011a, ApJ, 736, 38

Kocevski, D. D., Lubin, L. M., Gal, R., et al. 2009a, ApJ, 690, 295

Kocevski, D. D., Lubin, L. M., Lemaux, B. C., et al. 2009b, ApJ, 700, 901

Kocevski, D. D., Lubin, L. M., Lemaux, B. C., et al. 2009c, ApJ, 703, L33

Kushino, A., Ishisaki, Y., Morita, U., et al. 2002, PASJ, 54, 327

Laird, E. S., Nandra, K., Pope, A., \& Scott, D. 2010, MNRAS, 401, 2763

Lee, J. H., Lee, M. G., \& Hwang, H. S. 2006, ApJ, 650, 148

Lemaux, B. C., Gal, R. R., Lubin, L. M., et al. 2011, arXiv:1108.5799

Lemaux, B. C., Lubin, L. M., Sawicki, M., et al. 2009, ApJ, 700, 20

Lemaux, B. C., Lubin, L. M., Shapley, A., et al. 2010, ApJ, 716, 970

Lin, L., Koo, D. C., Weiner, B. J., et al. 2007, ApJ, 660, L51
Lubin, L. M., Brunner, R., Metzger, M. R., Postman, M., \& Oke, J. B. 2000, ApJ, 531, L5

Lubin, L. M., Gal, R. R., Lemaux, B. C., Kocevski, D. D., \& Squires, G. K. 2009, AJ, 137, 4867

Lubin, L. M., Mulchaey, J. S., \& Postman, M. 2004, ApJ, 601, L9

Lubin, L. M., Oke, J. B., \& Postman, M. 2002, AJ, 124, 1905

Lubin, L. M., Postman, M., \& Oke, J. B. 1998, AJ, 116, 643

Mann, R. G., Oliver, S., Carballo, R., et al. 2002, MNRAS, 332, 549

Mann, R. G., Oliver, S. J., Serjeant, S. B. G., et al. 1997, MNRAS, 289, 482

Marconi, A., \& Hunt, L. K. 2003, ApJ, 59, L21

Marconi, A., Risaliti, G., Gilli, R., et al. 2004, MNRAS, 351, 169

Martel, A. R., Menanteau, F., Tozzi, P., Ford, H. C., \& Infante, L. 2007, ApJS, 168,19

Martini, P., Mulchaey, J. S., \& Kelson, D. D. 2007, ApJ, 664, 761

McLean, I. S., Becklin, E. E., Bendiksen, O., et al. 1998, Proc. SPIE, 3354, 566

McNamara, B. R., \& Nulsen, P. E. J. 2007, ARA\&A, 45, 117

Menanteau, F., Jimenez, R., \& Matteucci, F. 2001, ApJ, 562, L23

Merloni, A., \& Heinz, S. 2008, MNRAS, 388, 1011

Mihos, J. C., \& Hernquist, L. 1996, ApJ, 464, 641

Murdoch, H. S., Crawford, D. F., \& Jauncey, D. L. 1973, ApJ, 183, 1

Nagao, T., Maiolino, R., \& Marconi, A. 2006, A\&A, 459, 85

Nandra, K., Georgakakis, A., Willmer, C. N. A., et al. 2007, ApJ, 660, L11

Oemler, A., Jr., Dressler, A., Kelson, D., et al. 2009, ApJ, 693, 152

Oke, J. B., Cohen, J. G., Carr, M., et al. 1995, PASP, 107, 375

Oke, J. B., Postman, M., \& Lubin, L. M. 1998, AJ, 116, 549

Pierce, C. M., Lotz, J. M., Laird, E. S., et al. 2007, ApJ, 660, L19

Poggianti, B. M., \& Barbaro, G. 1997, A\&A, 325, 1025

Poggianti, B. M., Smail, I., Dressler, A., et al. 1999, ApJ, 518, 576

Postman, M., Lubin, L. M., \& Oke, J. B. 1998, AJ, 116, 560

Postman, M., Lubin, L. M., \& Oke, J. B. 2001, AJ, 122, 1125

Rieke, G. H., Young, E. T., Engelbracht, C. W., et al. 2004, ApJS, 154, 25

Rosario, D. J., Mozena, M., Wuyts, S., et al. 2011, arXiv:1110.3816

Rosati, P., Tozzi, P., Giacconi, R., et al. 2002, ApJ, 566, 667

Rose, J. A. 1985, AJ, 90, 1927

Rutledge, R. E., Brunner, R. J., Prince, T. A., \& Lonsdale, C. 2000, ApJS, 131, 335

Sánchez, S. F., Becker, T., Garcia-Lorenzo, B., et al. 2005, A\&A, 429, L21

Sánchez, S. F., Jahnke, K., Wisotzki, L., et al. 2004, ApJ, 614, 586

Schawinski, K., Thomas, D., Sarzi, M., et al. 2007, MNRAS, 382, 1415

Schawinski, K., Virani, S., Simmons, B., et al. 2009, ApJ, 692, L19

Schiavon, R. P., Faber, S. M., Konidaris, N., et al. 2006, ApJ, 651, L93

Shields, G. A., Gebhardt, K., Salviander, S., et al. 2003, ApJ, 583, 124

Silverman, J. D., Kovač, K., Knobel, C., et al. 2009a, ApJ, 695, 171

Silverman, J. D., Lamareille, F., Maier, C., et al. 2009b, ApJ, 696, 396

Silverman, J. D., Mainieri, V., Lehmer, B. D., et al. 2008, ApJ, 675, 1025

Simcoe, R. A., Metzger, M. R., Small, T. A., \& Araya, G. 2000, BAAS, 32, 758

Somerville, R. S., Hopkins, P. F., Cox, T. J., Robertson, B. E., \& Hernquist, L. 2008, MNRAS, 391, 481

Springel, V., Di Matteo, T., \& Hernquist, L. 2005, MNRAS, 361, 776

Stott, J. P., Pimbblet, K. A., Edge, A. C., Smith, G. P., \& Wardlow, J. L. 2009, MNRAS, 394, 2098

Strateva, I., Ivezic, Z., Knapp, G. R., et al. 2001, AJ, 122, 1861

Sutherland, W., \& Saunders, W. 1992, MNRAS, 259, 413

Taylor, E. L., Mann, R. G., Efstathiou, A. N., et al. 2005, MNRAS, 361, 1352

Tozzi, P., Rosati, P., Nonino, M., et al. 2001, ApJ, 562, 42

Tremaine, S., Gebhardt, K., Bender, R., et al. 2002, ApJ, 574, 740

Trouille, L., Barger, A. J., \& Tremonti, C. 2011, ApJ, 742, 46

Weiner, B. J., Phillips, A. C., Faber, S. M., et al. 2005, ApJ, 620, 595

Wild, V., Heckman, T., \& Charlot, S. 2010, MNRAS, 405, 933

Xue, Y. Q., Brandt, W. N., Luo, B., et al. 2010, ApJ, 720, 368

Yan, R., Newman, J. A., Faber, S. M., et al. 2006, ApJ, 648, 281 